\title{
Dimensional reduction and gauge group reduction in Bianchi-type cosmology
}

\author{
J. M. Pons* \\ Departament d'Estructura i Constituents de la Matèria, Universitat de Barcelona, and Institut de Física d'Altes Energies, \\ Diagonal 647, E-08028 Barcelona, Catalonia, Spain \\ L. C. Shepley ${ }^{\dagger}$ \\ Center for Relativity, Physics Department, The University of Texas, Austin, Texas 78712-1081
}

(Received 6 February 1998; published 5 June 1998)

\begin{abstract}
In this paper we examine in detail the implementation, with its associated difficulties, of the Killing conditions and gauge fixing into the variational principle formulation of Bianchi-type cosmologies. We address problems raised in the literature concerning the Lagrangian and the Hamiltonian formulations: We prove their equivalence, make clear the role of the homogeneity preserving diffeomorphisms in the phase space approach, and show that the number of physical degrees of freedom is the same in the Hamiltonian and Lagrangian formulations. Residual gauge transformations play an important role in our approach, and we suggest that Poincaré transformations for special relativistic systems can be understood as residual gauge transformations. In the Appendixes, we give the general computation of the equations of motion and the Lagrangian for any Bianchi-type vacuum metric and for spatially homogeneous Maxwell fields in a nondynamical background (with zero currents). We also illustrate our counting of degrees of freedom in an appendix. [S0556-2821(98)04214-3]
\end{abstract}

PACS number(s): 04.20.Fy, 11.10.Ef, 98.80.Hw

\section{INTRODUCTION}

A spatially homogeneous cosmological model is a manifold $\mathcal{M}$ with a Lorentzian metric tensor $\mathbf{g}$ invariant under a group of isometries whose three-dimensional, spacelike, invariant hypersurfaces foliate $\mathcal{M}$. In the models we will treat, this group is generated by three spacelike vector fields $\mathbf{K}_{a}$, which span a Lie algebra defined by their commutation relations:

$$
\left[\mathbf{K}_{a}, \mathbf{K}_{b}\right]=C_{a b}^{c} \mathbf{K}_{c} .
$$

The invariance of the metric is expressed by the vanishing of its Lie derivatives with respect to these vectors: $\mathcal{L}_{\mathbf{K}_{a}} \mathbf{g}=0$. The structure constants are antisymmetric in their lower indices and obey the Jacobi relation

$$
C_{a b}^{c}=-C_{b a}^{c}, \quad C_{b e}^{a} C_{c d}^{e}+C_{c e}^{a} C_{d b}^{e}+C_{d e}^{a} C_{b c}^{e}=0 .
$$

The Bianchi classification of these algebras into nine types (see [1]) is the source of the term Bianchi-type cosmology. In three dimensions the Jacobi relation is equivalent to

$$
C_{e a}^{a} C_{b c}^{e}=0 .
$$

The algebras with vanishing $C_{e a}^{a}$ are called class $\mathrm{A}$ and those with nonvanishing $C_{e a}^{a}$ are called class B [2].

In a suitable basis, $\mathbf{g}$ can be represented by components $g_{\mu \nu}$ which depend only on a single variable, the cosmic time $t$. The Einstein field equations are (coupled, non-linear) ordinary equations. (The choice of this basis is part of this

\footnotetext{
*Electronic address: pons@ecm.ub.es

${ }^{\dagger}$ Electronic address: larry@helmholtz.ph.utexas.edu
}

paper.) The Einstein-Hilbert Lagrangian density for the metric may be computed in terms of these components and their $t$-derivatives. It is well known that in class A models this reduced Lagrangian does correctly reproduce the field equations, but it does not necessarily do so in class B models [3-7] because of a spatial divergence that is automatically zero only in class A [8].

Bianchi-type cosmology is still a subject of debate and-we think-some misunderstanding. It has been claimed, surprisingly, that the numbers of degrees of freedom in the Lagrangian and Hamiltonian formulations of these models do not agree [9]. This result would mean that the two formulations are not physically equivalent. Another issue, related to this one, poses the question as to what is to be considered a gauge transformation, and therefore a redundancy in the physical description in these models.

In this paper, we will address three subjects: the Lagrangian and Hamiltonian formulations of Bianchi-type models (dimensional reduction), the concept of gauge freedom for these models, and the fact that the numbers of degrees of freedom in the Lagrangian and Hamiltonian formulations do agree. We do not solve the first subject, which remains a problem for class B models, although we clarify some points that are also relevant for class A models. From our analysis we give an answer to the second and third problems. We show that the gauge freedom is dictated by the diffeomorphism invariance of the original theory and leads to the physical equivalence of the Lagrangian and Hamiltonian formulations.

We treat homogeneous models in general relativity as constrained dynamical systems. In particular we clarify the two aspects that merge when we move from the superspace of all metrics to the minisuperspace of homogeneous metrics. On the one hand, there is the dimensional reduction from $3+1$ spacetime dimensions to one time dimension. This di- 
mensional reduction comes from the symmetry associated with the Lie algebra of the Killing vectors. On the other hand, there is the elimination of gauge degrees of freedom. These two types of reduction present different types of problems, which we analyze, but both are necessary in order to reduce the original gauge group of four-diffeomorphism invariance to the gauge group of time reparametrization invariance.

This reduction procedure will be undertaken in four steps. As an outcome of our analysis, (a) we will clarify some points concerning the Lagrangian and Hamiltonian reduced formulations, (b) we will be able to point out clearly what the gauge group is for these models, and (c) we will show that the correct reduction procedures in both the Lagrangian and Hamiltonian formulations lead to a number of degrees of freedom which is always the same in either formulation. The first step, in Sec. II, consists of adapting time coordinates to the symmetry group. The second step, in Sec. III, is to adapt spatial coordinates to the group by adopting a gauge in which the shift vector depends only on time. The third step, in Sec. IV, is to adopt a gauge in which the shift vector vanishes. Finally, the fourth step, in Sec. V, is to eliminate residual three-diffeomorphisms. After each step we examine the status of what remains of the gauge group. In Sec. VI we examine the loss of constraints caused by the gauge fixing. We perform, in Sec. VII, the Hamiltonian analysis, which is presented in a summary form because it is parallel to the Lagrangian analysis. Section VIII is devoted to conclusions.

In Appendix A we present explicit equations for spatially homogeneous metrics in full detail, including a general lapse function and shift vector, for any Bianchi type, where coordinates have been chosen so that all variables depend only on time. The purpose is to provide concrete examples for our formalism. A simpler example, which also can serve to illustrate our methods, is provided in Appendix B: It is the case of a spatially homogeneous electromagnetic field in a spatially homogeneous background metric. Appendix $\mathrm{C}$ reviews the general literature on the relationship between setting gauge conditions and variational principles. It gives justification to some results used in Secs. IV and VII. Appendix D illustrates our counting of degrees of freedom in the Bianchi type I case and shows how spacetime rigid symmetries may be considered as residual gauge transformations.

\section{TIME}

We start with the general setting for Bianchi models: Call $T$ the set, each element of which is a metric tensor $\mathbf{g}$ and three vector fields $\mathbf{K}_{a}$ whose orbits generate threedimensional hypersurfaces which foliate the fourdimensional manifold $\mathcal{M}$; the Lie derivatives of $\mathbf{g}$ with respect to the $\mathbf{K}_{a}$ vanish. The invariance of $\mathbf{g}$ and even the definition of the $\mathbf{K}_{a}$ need only be locally defined for much of what we do; if the vector fields are globally defined, we speak of global homogeneous cosmologies [9].

The diffeomorphisms on $\mathcal{M}$ can be viewed in an active sense, mapping points onto other points. In that case, an element (metric plus three vector fields) of $\mathrm{T}$ will in general be mapped to another element in T. If there is a covering of
$\mathcal{M}$ by coordinate patches, each diffeomorphism may also be viewed in a passive sense as a collection of coordinate transformations. It is this passive sense which is closer to the physical principle which is a motivation for general relativity, that physics should not depend on coordinates. It is, in fact, somewhat easier to adopt the language of a metric being determined by its components in a coordinate patch; a diffeomorphism is then a transformation which preserves the metric $\mathbf{g}$ and the vectors $\mathbf{K}_{a}$ but in general changes their components $g_{\mu \nu}, K_{a}^{\mu}$. Nevertheless, we shall adopt the active view when convenient. (Greek indices range over $0,1,2,3$, with the coordinate $x^{0}$ being the time $t$. Latin indices will range over $1,2,3$.)

Given a coordinate neighborhood $\mathcal{N}$, we consider all metric tensors, defined by their components $g_{\mu \nu}$ in $\mathcal{N}$, which are invariant under isometries defined by the $\mathbf{K}_{a}$ and in which the $\mathbf{K}_{a}$ are spacelike. The metric components satisfy the Killing equation

$$
0=\left(\mathcal{L}_{\mathbf{K}_{a}} \mathbf{g}\right)_{\mu \nu}=K_{a}^{\sigma} g_{\mu \nu, \sigma}+g_{\sigma \nu} K_{a, \mu}^{\sigma}+g_{\mu \sigma} K_{a, \nu}^{\sigma},
$$

where a comma denotes partial differentiation. We can consider $\mathrm{T}$ as being the collection of all such tensor components, each element of $T$ being the collection of metric and Killing vector components, the coordinate system being understood. Any of the elements of $\mathbb{T}$ foliates $\mathcal{M}$ by three-dimensional space-like homogeneous hypersurfaces, namely the integral surfaces of the Killing vector fields.

A change of coordinates-a diffeomorphism-will in general change the form of the metric components. In that sense, the diffeomorphism group $\operatorname{Diff}(\mathcal{M})$ on $\mathcal{M}$ is realized as a group $\mathbf{D}$ acting on $T$. The physics does not change, of course, under a coordinate transformation, and symbolically we can write

$$
\text { physics }(\text { of a Bianchi type })=T / \mathbf{D} \text {. }
$$

These conditions need only hold locally for much of what we will be doing. In that sense, we really are working with a Lie algebra of infinitesimal isometries rather than a Lie group, though we will continue to speak of the isometries as a symmetry group. When we need to require global homogeneity, we will clearly specify so.

The existence of these isometries of the metric can be used to simplify greatly the equations of motion, which we take to be the vacuum Einstein equations. At this point, the full gauge group D of general relativity, generated by Diff $\mathcal{M}$, is still operating in $\mathbb{T}$. We will be looking for a reduced Lagrangian, with the Killing conditions built in, capable of describing these Bianchi models. We do so in four steps: adapting time, adapting space, fixing the gauge by requiring the shift vector (to be defined later) to vanish, and fixing the residual gauge freedom.

We now proceed to the first step, which will partially fix the gauge (that is, fixing coordinates) by concentrating on the three-dimensional homogeneous hypersurfaces. Suppose that the homogeneous hypersurfaces happen to coincide with the hypersurfaces $t=$ const in the coordinates defining an element of $\mathrm{T}\left(g_{\mu \nu}, K_{a}^{\sigma}\right)$; then we introduce an equivalence re- 
lation by saying that another element of $T$ is equivalent to this one if it is produced by an element of $\mathbf{D}$, that is, by a diffeomorphism. The set of these equivalence classes is $\mathbb{T}_{t}$, and the full group $\mathbf{D}$ has been reduced to a smaller group $\mathbf{D}_{t}$, namely those diffeomorphisms which preserve the condition that the homogeneous hypersurfaces be defined by $t=$ const.

In this first step, we have made a choice of spacetime local coordinates such that the surfaces $\Sigma_{t}$ of constant time coincide with the foliation defined by the Killing vectors. The time coordinate is a function whose curl vanishes under Lie differentiation by any of the Killing vectors:

$$
\mathcal{L}_{\mathbf{K}_{a}} \mathbf{d} t=0 .
$$

Our Killing vectors now take the general form (where $\left.\boldsymbol{\partial}_{i} \equiv \partial / \partial x^{i}\right)$

$$
\mathbf{K}_{a}=K_{a}^{i}(t, x) \boldsymbol{\partial}_{i} .
$$

Notice the possible time dependence of $\mathbf{K}_{a}$. (We denote the four spacetime coordinates $t, x^{i}$ by $t, x$ when they are used as the arguments of a function.)

\section{A. Gauge group after the first step}

In these spacetime coordinates $\left\{t, x^{i}\right\}$, a general infinitesimal reparametrization (coordinate transformation) is generated by a vector field $\epsilon^{\mu}(x) \boldsymbol{\partial}_{\mu}$ :

$$
\left(\begin{array}{c}
t \\
x^{i}
\end{array}\right) \rightarrow\left(\begin{array}{c}
t+\delta t \\
x^{i}+\delta x^{i}
\end{array}\right)=\left(\begin{array}{c}
t+\epsilon^{0} \\
x^{i}+\epsilon^{i}
\end{array}\right) .
$$

To preserve Eq. (2.2), that is, to generate a foliationpreserving diffeomorphism, requires

$$
\epsilon_{, i}^{0}=0 .
$$

\section{B. Expressing the homogeneous metric}

Recall the commutation relations of the Killing vector equation (1.1). At this point it is customary to introduce a set of three independent, right-invariant (under the Lie algebra) vector fields $\mathbf{Y}_{a}=Y_{a}^{i}(t, x) \boldsymbol{\partial}_{i}$, which satisfy

$$
\left[\mathbf{K}_{a}, \mathbf{Y}_{b}\right]=0 .
$$

We take them to be tangent to the homogeneous hypersurfaces. They also define a Lie algebra and can be taken such that

$$
\left[\mathbf{Y}_{a}, \mathbf{Y}_{b}\right]=-C_{a b}^{c} \mathbf{Y}_{c} .
$$

These commutation relations are ensured if we take $\mathbf{Y}_{a}$ to coincide with $\mathbf{K}_{a}$ at an arbitrary point in $\Sigma_{t}$ and define them at other points in $\Sigma_{t}$ by Eq. (2.6) [1].

In each $\Sigma_{t}$ we can define a basis of 1-forms, $\boldsymbol{\omega}^{a}=\omega_{i}^{a}(t, x) \mathbf{d} x^{i}$, dual to these left-invariant vectors: $\boldsymbol{\omega}^{a}\left(\mathbf{Y}_{b}\right)=\delta_{b}^{a}$. The Lie algebra property becomes $\left(\mathbf{d}_{3}\right.$ is differentiation with respect to the space variables)

$$
\mathbf{d}_{3} \boldsymbol{\omega}^{a}=\frac{1}{2} C_{b c}^{a} \boldsymbol{\omega}^{b} \wedge \boldsymbol{\omega}^{c}
$$

The metric can be written, using the anholonomic basis $\left\{\mathbf{d} t, \boldsymbol{\omega}^{a}\right\}$, as

$$
\mathbf{g}=-N^{2} \mathbf{d} t^{2}+g_{a b}\left(N^{a} \mathbf{d} t+\boldsymbol{\omega}^{a}\right)\left(N^{b} \mathbf{d} t+\boldsymbol{\omega}^{b}\right),
$$

where $N$ is the lapse function and $N^{b}$ the shift vector variables in this basis. The Killing conditions on g give (an overdot denotes $\partial / \partial t$ )

$$
\begin{gathered}
\mathbf{K}_{a}\left(g_{b c}\right)=0 \Leftrightarrow g_{a b}=g_{a b}(t), \\
\mathbf{K}_{a}(N)=0 \Leftrightarrow N=N(t), \\
\mathcal{L}_{\mathbf{K}_{a}}\left(N^{b} \mathbf{d} t+\boldsymbol{\omega}^{b}\right)=0 \Leftrightarrow \dot{\mathbf{K}}_{a}=\left[N^{b} \mathbf{Y}_{b}, \mathbf{K}_{a}\right]=-\mathbf{K}_{a}\left(N^{b}\right) \mathbf{Y}_{b} .
\end{gathered}
$$

The first two results are clear: no spatial dependence for $N$ and $g_{a b}$. The third result links the time dependence of the Killing vectors to the spatial dependence of the shift variables $N^{a}$. It relates dimensional reduction and gauge-fixing reduction. Since the shift variables are completely arbitrary because of the gauge freedom, namely diffeomorphisms satisfying $\epsilon_{, i}^{0}=0$ [see Eq. (2.5)], we may consider this third relation as giving the time evolution of the Killing vector components for a given set of the shift variables. This shows in particular that if for an "initial" time, say $t=0$, the Killing conditions are satisfied, they will be satisfied in future times, the third relation providing us with the form which the Killing vectors take.

At this point we could proceed to reduce the Lagrangian (which we will describe below). But reduction within the Lagrangian and reduction in the equations of motion are procedures that in general do not commute, as we could immediately verify. In the next section a thorough study of this non-commutativity will be given.

The fact that the shift variables depend arbitrarily on the space coordinates shows that we must further reduce the gauge freedom in order to end up with the reduced gauge group of time-reparametrization invariance. To do so, the simplest way is to require the shift variables to be spatially constant. According to the third relation above, this is equivalent to requiring time independence of the Killing vectors. This is our second step in the gauge fixing procedure, in the next section, and is a case of adapting spatial coordinates to the Killing structure.

\section{SPACE}

We introduce the partial gauge-fixing conditions

$$
N_{, i}^{a}=0 .
$$

We know from the previous analysis, Eq. (2.10c), that the Killing vectors become time independent,

$$
\mathbf{K}_{a}=K_{a}^{i}(x) \boldsymbol{\partial}_{i},
$$


and we can also choose the right invariant vectors $\mathbf{Y}_{a}$ and the 1 -forms $\boldsymbol{\omega}^{a}$ to be so. It was realized in [10] that the shift vector $\mathbf{N} \equiv N^{a}(t) \mathbf{Y}_{a}$ is a one-parameter family of inner automorphisms of the right-invariant Lie algebra (generators $\mathbf{Y}_{b}$ ). More details are given in Sec. V. Now the metric is written as

$$
\mathbf{g}=-N^{2}(t) \mathbf{d} t^{2}+g_{a b}(t)\left[N^{a}(t) \mathbf{d} t+\boldsymbol{\omega}^{a}\right]\left[N^{b}(t) \mathbf{d} t+\boldsymbol{\omega}^{b}\right],
$$

with

$$
\boldsymbol{d} \boldsymbol{\omega}=\frac{1}{2} C_{b c}^{a} \boldsymbol{\omega}^{b} \wedge \boldsymbol{\omega}^{c},
$$

and the Killing conditions are built in.

We proceed to study the non-commutativity of the operations of introducing the conditions $g_{a b, i}=N_{, i}=N_{, i}^{a}=0$ into the equations of motion (that is, to look for a restricted set of solutions of the Einstein equations) or directly into the Lagrangian. The calculation given in Appendix A shows explicit examples of the ideas we discuss in this section. In the anholonomic basis $\left\{\mathbf{d} t, \boldsymbol{\omega}^{a}\right\}$, the Einstein-Hilbert Lagrangian density, once a total divergence is discarded, can be written as (our notation follows [11])

$$
\mathcal{L}=|\omega| \sqrt{g} N\left({ }^{3} R+K_{a b} K^{a b}-K^{2}\right) \equiv|\omega| \widetilde{\mathcal{L}},
$$

where $|\omega|=\operatorname{det}\left(\omega_{i}^{a}\right), g=\operatorname{det}\left(g_{a b}\right), N \neq 0$ is the lapse function, ${ }^{3} R$ is the three-metric curvature, $K_{a b}$ the extrinsic curvature,

$$
K_{a b}=\frac{1}{2 N}\left(\dot{g}_{a b}-N_{a \mid b}-N_{b \mid a}\right),
$$

and $K=e^{a b} K_{a b}$ its trace (the vertical bar denotes the spatial covariant derivative and $e^{a b}$ is the matrix inverse of $g_{a b}$ ). Notice that $\mathcal{L}$ is second order in the three-space derivatives.

One could also add other fields in the Lagrangian and implement the Killing conditions on them. For instance, in the Einstein-Maxwell case, the one-form gauge field $\mathbf{A}=A_{0} \mathbf{d} t+A_{a} \boldsymbol{\omega}^{a}$ must satisfy $A_{0, i}=A_{a, i}=0$ (see Appendix B). If we label as $X$ the generic variable $g_{a b}, N, N^{a}, A_{0}, A_{a}, \ldots, \quad$ then $\quad \widetilde{\mathcal{L}} \quad$ is a function $\widetilde{\mathcal{L}}\left(X, \dot{X}, \mathbf{Y}_{b} X, \mathbf{Y}_{a} \mathbf{Y}_{b} X\right)$, where the last argument in $\widetilde{\mathcal{L}}$ has $a \geqslant b$. Notice that $\mathbf{Y}_{a} \mathbf{Y}_{b} X$ contains not only second order but also first order spatial derivatives of $X$.

Let us write the Euler-Lagrange equations using the variables just displayed. The variation $\delta \mathcal{L}$ is

$$
\begin{aligned}
\delta \mathcal{L}= & \frac{\partial \mathcal{L}}{\partial X} \delta X+\frac{\partial \mathcal{L}}{\partial \dot{X}} \delta \dot{X}+\frac{\partial \mathcal{L}}{\partial \mathbf{Y}_{a} X} \delta\left(\mathbf{Y}_{a} X\right) \\
& +\sum_{a \geqslant b} \frac{\partial \mathcal{L}}{\partial \mathbf{Y}_{a} \mathbf{Y}_{b} X} \delta\left(\mathbf{Y}_{a} \mathbf{Y}_{b} X\right) .
\end{aligned}
$$

Integration by parts, dropping the boundary terms, and repeated use of the relation

$$
\mathbf{Y}_{a}(|\omega|)+|\omega| \boldsymbol{\partial}_{j} Y_{a}^{j}=|\omega| C_{a d}^{d}
$$

yields the following form for the Euler-Lagrange equations:

$$
\begin{aligned}
\frac{\delta \mathcal{L}}{\delta X}=\mid & \omega \mid\left[\frac{\partial \widetilde{\mathcal{L}}}{\partial X}-\partial_{t} \frac{\partial \widetilde{\mathcal{L}}}{\partial \dot{X}}-\left(\mathbf{Y}_{a}+C_{a c}^{c}\right)\left(\frac{\partial \widetilde{\mathcal{L}}}{\partial \mathbf{Y}_{a} X}\right)\right. \\
& \left.+\sum_{a \geqslant b}\left(\mathbf{Y}_{b}+C_{b d}^{d}\right)\left(\mathbf{Y}_{a}+C_{a c}^{c}\right)\left(\frac{\partial \widetilde{\mathcal{L}}}{\partial \mathbf{Y}_{a} \mathbf{Y}_{b} X}\right)\right] .
\end{aligned}
$$

Because of the particular structure of Eq. (3.3), the third and fourth order spacetime derivatives in Eq. (3.6) will cancel, but this fact does not alter our discussion.

If we define the reduced Lagrangian

$$
\mathcal{L}_{R}(X, \dot{X}) \equiv \widetilde{\mathcal{L}}\left(X, \dot{X}, \mathbf{Y}_{a} X=0, \mathbf{Y}_{a} \mathbf{Y}_{b} X=0\right),
$$

we end up with

$$
\begin{aligned}
\left(\frac{\delta \mathcal{L}}{\delta X}\right)_{\left(\partial_{i} X=\partial_{i j} X=0\right)}=\mid & \omega \mid\left[\frac{\delta \mathcal{L}_{R}}{\delta X}-C_{a c}^{c}\left(\frac{\partial \widetilde{\mathcal{L}}}{\partial \mathbf{Y}_{a} X}\right)_{\left(\partial_{i} X=\partial_{i j} X=0\right)}\right. \\
& \left.+\sum_{a \geqslant b} C_{a c}^{c} C_{b d}^{d}\left(\frac{\partial \widetilde{\mathcal{L}}}{\partial \mathbf{Y}_{a} \mathbf{Y}_{b} X}\right)_{\left(\partial_{i} X=\partial_{i j} X=0\right)}\right] .
\end{aligned}
$$

Equation (3.7) displays the non-commutativity between the two procedures: reduction of the Lagrangian through the Killing conditions or reduction of the equations of motion. As we have mentioned before, this problem was identified long ago. The distinction between class A and class B Bianchi models is crucial in this respect, for class A is characterized by the vanishing of the trace $C_{a b}^{b}$ [2].

Summing up, we see that the implementation of the Killing conditions within the Lagrangian produces no harm; that is, commutativity holds in Eq. (3.7), for class A models. For class B models the equations of motion derived from $\mathcal{L}_{R}$ are wrong when there is a contribution different from zero coming from the last two pieces in Eq. (3.7). One may wonder whether this means that the situation is hopeless if we want to have a Lagrangian formulation for class B models. Perhaps in general, but in some special cases there may be $a d$ hoc solutions. In Appendix B we show an example of such a case within homogeneous Maxwell theory.

At this stage we have time-independent Killing vectors. Let $\mathbf{E}=\epsilon^{\mu} \boldsymbol{\partial}_{\mu}=\epsilon^{0} \boldsymbol{\partial}_{0}+\epsilon^{a} Y_{a}^{i} \boldsymbol{\partial}_{i}$ be a generator of a diffeomorphism which preserves this requirement as well as Eq. (2.5):

$$
\left[\mathbf{E}, \mathbf{K}_{a}\right]^{\cdot}=\left[\dot{\mathbf{E}}, \mathbf{K}_{a}\right]=0, \quad \epsilon^{0}=\epsilon^{0}(t),
$$

where $=\partial / \partial t$. The general form for the spatial components of $\mathbf{E}$ is given by

$$
\epsilon^{a}(t, x)=\varphi^{a}(t)+\zeta^{a}(x),
$$

with $\varphi^{a}(t)$ and $\zeta^{i}(x)$ arbitrary functions of time and spatial coordinates respectively. 

(3.8):

Suppose $\mathbf{E}_{1}$ and $\mathbf{E}_{1}$ are two generators satisfying Eq.

$$
\left[\dot{\mathbf{E}}_{1}, \mathbf{K}_{a}\right]=\left[\dot{\mathbf{E}}_{2}, \mathbf{K}_{a}\right]=0
$$

By the Jacobi identity it is clear that $\left[\left[\dot{\mathbf{E}}_{1}, \dot{\mathbf{E}}_{2}\right], \mathbf{K}_{a}\right]=0$, but it is not necessarily the case that $\left.\left[\mathbf{E}_{1}, \mathbf{E}_{2}\right]^{\circ}, \mathbf{K}_{a}\right]=0$. A closure process which is more general than commutation applies for these generators. The infinitesimal diffeomorphism generated by $\mathbf{E}_{1}$ changes the Killing vector fields (in the active view of diffeomorphisms), but it also changes the invariant basis vector fields and the second generator $\mathbf{E}_{2}$ as well. These changes, particularly changes in the invariant basis vectors $\mathbf{Y}_{a}$, mean that the form of Eq. (3.9) cannot be expected to be invariant during the process of commutation. We will not pursue this matter further, for in the next step, where the shift vector is set to zero, the form of the gauge group once again becomes straightforward.

Notice that for class A models, the first term on the right in Eq. (3.9) is a Noether symmetry for $\mathcal{L}_{R}$. Our Lagrangian $\mathcal{L}$, Eq. (3.3), differs from the Einstein-Hilbert Lagrangian $\mathcal{L}_{E H}$ by a divergence

$$
\mathcal{L}=\mathcal{L}_{E H}+|\omega| \sqrt{g} m_{\mid a}^{a} .
$$

General reparametrization invariance under a diffeomorphism generated by $\mathbf{v}=\epsilon^{\mu}(x) \boldsymbol{\partial}_{\mu}=\varphi^{a}(t) \mathbf{Y}_{a}$ produces the functional variation of $\mathcal{L}_{E H}$ :

$$
\delta_{\mathbf{v}} \mathcal{L}_{E H}=\boldsymbol{\partial}_{\mu}\left(\epsilon^{\mu} \mathcal{L}_{E H}\right) .
$$

Therefore,

$$
\delta_{\mathbf{v}} \mathcal{L}=\boldsymbol{\partial}_{\mu}\left(\epsilon^{\mu} \mathcal{L}_{E H}\right)+\delta_{\mathbf{v}}\left(|\omega| \sqrt{g}\left(\mathbf{Y}_{a} m^{a}+C_{a b}^{b} m^{a}\right)\right)
$$

Let us now reduce these expressions, for class A models, introducing our second step gauge fixing, $\boldsymbol{\partial}_{i} X=\boldsymbol{\partial}_{i j} X=0$, where $X=g_{a b}, N, N^{a}, A_{0}, A_{a}, \ldots$. The left side of Eq. (3.10) becomes

$$
|\omega| \delta_{\mathbf{v}} \mathcal{L}_{R}
$$

The first term on the right in Eq. (3.10) is

$$
\left(\boldsymbol{\partial}_{\mu}\left(\epsilon^{\mu} \mathcal{L}\right)\right)_{\left(\partial_{i} X=\partial_{i j} X=0\right)}=f^{a}(t) \boldsymbol{\partial}_{i}\left(|\omega| Y_{a}^{i} \widetilde{\mathcal{L}}\right)_{\left(\partial_{i} X=\partial_{i j} X=0\right)}=0
$$

where we have used Eq. (3.5) in the last step. The second term on the right in Eq. (3.10) becomes

$$
\begin{aligned}
\left(\delta_{\mathbf{v}}\left(|\omega| \sqrt{g} m_{\mid a}^{a}\right)\right)_{\left(\partial_{i} X=\partial_{i j} X=0\right)} & =|\omega| \mathbf{Y}_{a}\left(\delta_{\mathbf{v}}\left(\sqrt{g} m^{a}\right)\right)_{\left(\partial_{i} X=\partial_{i j} X=0\right)} \\
& =0 .
\end{aligned}
$$

We end up with $\delta_{\mathbf{v}} \mathcal{L}_{R}=0$; this is a gauge Noether symmetry provided by the reduced theory.

The second term on the right in Eq. (3.9) is not retrievable as a gauge symmetry from the reduced Lagrangian formulation (except when it is a copy, with constants $\varphi^{a}$, of the first term). However, we must still provide a gauge fixing for it. In step 4 of our gauge fixing procedure we will deal with it.
It is obvious from Eq. (3.9) that the process of reducing the gauge group is not yet finished; we now continue this task.

\section{ZERO SHIFT}

The remaining diffeomorphism invariance allows us to perform a new partial gauge fixing by introducing a set of constraints that helps to eliminate some of the arbitrariness that exists in our equations of motion. To simplify the analysis we consider the case in which the metric is the only field in $\mathcal{L}$. Should other gauge fields be present, the dynamics could have additional arbitrariness.

Notice that the Lagrangian is independent of $\dot{N}$ and $\dot{N}^{a}$. This fact implies that the dynamical evolution vector field operator in configuration-velocity space has $[12,13]$ a term of the type (see Appendix $\mathrm{C}$ for clarification of this point)

$$
\int d^{3} x\left(\eta^{0}(t, x) \frac{\partial}{\partial \dot{N}}+\eta^{a}(t, x) \frac{\partial}{\partial \dot{N}^{a}}\right),
$$

with $\eta^{0}, \eta^{a}$ arbitrary functions. The important consequence of this arbitrariness is that to fix the dynamics we must fix the values of $N$ and $N^{a}$. Here we are only interested in a partial gauge fixing: We only fix the shift functions. The simplest way of doing so is by introducing the gauge-fixing constraints $N^{a}=0$. It is always possible to pass from an initial configuration with $N^{a} \neq 0$ to a final configuration with $N^{a}=0$ by using diffeomorphisms satisfying the restrictions of Eq. (3.9); the geometric picture of this transformation is to make the curves in $\mathcal{M}$ tangent to the normal vector of the $\Sigma_{t}$ coincident with the curves generated by $\boldsymbol{\partial}_{t}$.

This is the Lagrangian version of the partial gauge fixing. Stability of the new constraints under time evolution yields the new constraints $\dot{N}^{a}=0$; then requiring stability again will make the arbitrary functions $\eta^{a}=0$.

To complete the picture, let us go back to the second gauge fixing step, Eq. (3.1). Consider Eq. (4.1) again. Stabilization of $N_{, i}^{a}=0$ implies $\dot{N}_{, i}^{a}=0$. The stabilization of $\dot{N}_{, i}^{a}=0$ yields $\eta_{, j}^{a}=0$. By the same token, $\eta_{, j}^{0}$ vanishes as a consequence of the relation, which is also a gauge fixing, $N_{, i}=0$, obtained in the first gauge fixing step. Therefore, from the point of view of the reduction of the equations of motion, the arbitrariness in the dynamical evolution vector field operator is described by four functions $\eta^{0}(t), \eta^{a}(t)$. The gauge freedom associated with this arbitrariness is given by $\epsilon^{0}(t), \varphi^{a}(t)$ in Eqs. (2.5) and (3.9). Notice that the second term in Eq. (3.9) is unretrievable from the reduced dynamics. This is another way to verify the limitations of the reduced formalism under the Killing conditions already pointed out in the last paragraphs of Sec. III.

Once the shift functions $N^{a}$ have been set to zero, the gauge group has been greatly reduced, for the only remaining diffeomorphisms still available to us are those that, besides keeping the three-foliation, preserve the conditions $N^{a}=0$. This requirement results in

$$
0=\dot{\epsilon}^{a}
$$


The remaining diffeomorphisms therefore are such that $\epsilon^{0}$ only depends on $t$ [see Eq. (2.5)], whereas the $\epsilon^{a}$ depend only on the three-space coordinates $x^{i}$ [see Eq. (4.2)]. A nice picture emerges: The remaining gauge group has been factorized into two commuting subgroups, the group of time reparametrizations and the group of three-space diffeomorphisms.

Properly speaking, only the first group is still a gauge group; it is directly associated with the freedom that is left in the Lagrangian evolution operator and that is displayed in the arbitrariness of $\eta^{0}$ in Eq. (4.1) ( $\eta^{a}$ are zero in our particular gauge fixing).

The group of three-space diffeomorphisms is not a gauge group because it does not have room for what is characteristic of the gauge freedom: to connect the members of a family of field configurations, all of which are solutions of the equations of motion, which share the same set of initialvalue conditions. This group must be understood as describing a redundancy in the space of initial conditions for our theories. We call this group a residual gauge group. A parallel case in electromagnetism is the residual gauge symmetry that is left after the introduction of the Lorentz gauge $\boldsymbol{\partial}_{\mu} A^{\mu}=0$ : The transformation $A_{\mu} \rightarrow A_{\mu}+\partial_{\mu} \Lambda$ is a residual gauge symmetry if $\square \Lambda=0$. In any case, one must take into account that the gauge fixing procedure is only finished when we have completely removed these residual gauge transformations [13]. Further comments on this important point are made in the last section of this paper.

We finish this section by observing that the comments raised in the previous section still apply here: Only the diffeomorphisms of the form $\epsilon^{i}=B^{a} \mathbf{Y}_{a}^{i}$, with $B^{a}$ constant, are obtainable as gauge Noether symmetries from the reduced Lagrangian in class A models. In the language introduced in the next section, these vector fields $B^{a} \mathbf{Y}_{a}^{i}$ define the inner automorphisms for the right invariant Lie algebra (generators $\left.\mathbf{Y}_{a}\right)$.

\section{RESIDUAL THREE-DIFFEOMORPHISMS}

Now we are ready to perform the fourth step of the gauge fixing. Notice that the three-metric $g_{a b}(t) \boldsymbol{\omega}^{a} \boldsymbol{\omega}^{b}$ on the surfaces $\Sigma_{t}$ is also Killing with respect to the vectors $\mathbf{K}_{a}$. We are going to fix, in principle, the residual threediffeomorphism group by working from now on with this basis of one-forms $\left\{\boldsymbol{\omega}^{a}\right\}$. In the active view, a general threediffeomorphism will drive $\mathbf{K}_{a} \rightarrow \mathbf{K}_{a}^{\prime}, \boldsymbol{\omega}^{a} \rightarrow \boldsymbol{\omega}^{\prime a}$. The transformed metric $g_{a b}(t) \boldsymbol{\omega}^{\prime a} \boldsymbol{\omega}^{\prime b} \equiv g_{a b}^{\prime}(t, x) \boldsymbol{\omega}^{a} \boldsymbol{\omega}^{b}$ will be Killing with respect to the new set $\mathbf{K}_{a}^{\prime}$ and will in general no longer be Killing with respect to the original vectors $\mathbf{K}_{a}$. In other words, $g_{a b}^{\prime}$ will in general acquire a dependence on the spatial coordinates. However, there is another possibility to explore: the case when the new set $\mathbf{K}_{a}^{\prime}$ happens to belong to the Lie algebra generated by the original Killing vectors $\mathbf{K}_{a}$. This is equivalent to saying that

$$
\boldsymbol{\omega}^{\prime a}=M_{b}^{a} \boldsymbol{\omega}^{b},
$$

with $M_{b}^{a}$ constant. These are the homogeneity-preserving diffeomorphisms (HPDs), which were introduced by Ashtekar and Samuel [9].

Generators $\mathbf{v}$ for the HPDs are better expressed in the basis of the invariant vectors $\mathbf{Y}_{a}, \mathbf{v}=f^{a}(x) \mathbf{Y}_{a}$. The condition for a HPD is ( $\mathcal{L}$ is Lie derivative here)

$$
\mathcal{L}_{\mathbf{v}} \boldsymbol{\omega}^{b}=B_{a}^{b} \boldsymbol{\omega}^{a},
$$

with $B_{a}^{b}$ a constant matrix. This is equivalent to

$$
\mathcal{L}_{\mathbf{v}} \mathbf{K}_{a}=-A_{a}^{b} \mathbf{K}_{b},
$$

with $A_{a}^{b}$ a constant matrix. The left-invariant Lie algebra (generators $\mathbf{K}_{a}$ ) and the right-invariant Lie algebra (generators $\mathbf{Y}_{a}$ ) possess the same automorphisms. If the point $x_{0}$ in $\Sigma$ is the point where $\mathbf{Y}_{a}$ coincides with $\mathbf{K}_{a}$, then $B_{a}^{b}=A_{a}^{b}+f^{c}\left(x_{0}\right) C_{c a}^{b}$.

Equation (5.3) is

$$
\left(\mathbf{K}_{a} f^{c}\right) \mathbf{Y}_{c}=-\left[\mathbf{v}, \mathbf{K}_{a}\right]=A_{a}^{b} \mathbf{K}_{b} \Leftrightarrow\left(\mathbf{K}_{a} f^{c}\right)=\left(\mathbf{K}_{b} \cdot \boldsymbol{\omega}^{c}\right) A_{a}^{b} .
$$

This last equation is equivalent to

$$
\mathbf{d} f^{c}=\left(\mathbf{K}_{b} \cdot \boldsymbol{\omega}^{c}\right) A_{a}^{b} \mathbf{K}^{a},
$$

where $\mathbf{K}^{a}$ are the dual forms to $\mathbf{K}_{b}$. Notice that $\left(\mathbf{K}_{b} \cdot \boldsymbol{\omega}^{c}\right)$ is the adjoint representation of the Lie group expressed in the local patch we are working in. Because $\mathbf{d} \mathbf{K}^{c}=-\frac{1}{2} C_{a b}^{c} \mathbf{K}^{a} \wedge \mathbf{K}^{b}$ and $\mathbf{d}\left(\mathbf{K}_{d} \cdot \boldsymbol{\omega}^{c}\right)=C_{b d}^{e}\left(\mathbf{K}_{e} \cdot \boldsymbol{\omega}^{c}\right) \mathbf{K}^{b}$, the local integrability conditions for Eq. (5.5),

$$
\mathbf{d}\left[\left(\mathbf{K}_{b} \cdot \omega^{c}\right) A_{b}^{a} \mathbf{K}^{a}\right]=0,
$$

read

$$
C_{e b}^{a} A_{c}^{e}-C_{e c}^{a} A_{b}^{e}+C_{b c}^{e} A_{e}^{a}=0,
$$

which are the conditions for Lie algebra automorphisms. Trivial solutions are $A_{b}^{a}=0$ and $A_{b}^{a}=A^{c} C_{c b}^{a}$, with $A^{c}$ constant. The solutions for the first case are

$$
\mathbf{v}=B^{c} \mathbf{Y}_{c},
$$

with $B^{c}$ constant, and for the second,

$$
\mathbf{v}=A^{c} \mathbf{K}_{c} .
$$

The first (second) solutions describe the inner automorphisms for the right-invariant (left-invariant) Lie algebra. In finite form, the $M_{b}^{a}$ in Eq. (5.1) $\left[\mathbf{M}=e^{\mathbf{B}}, \mathbf{B}\right.$ of Eq. (5.2)] satisfy

$$
\left(\mathbf{M}^{-1}\right)_{a}^{d}\left(\mathbf{M}^{-1}\right)_{b}^{e} C_{d e}^{f} M_{f}^{c}=C_{a b}^{c} .
$$

The integrability conditions, Eq. (5.7), only guarantee the local existence of the HPDs. But we need these HPDs to be global diffeomorphisms if Eq. (5.10) is to hold everywhere. Both the Lie algebra structure and the topology of the surfaces of homogeneity play a role in the determination of the existing HPDs. In particular, for globally homogeneous cos- 
mologies with simply connected surfaces of homogeneity, every solution of Eq. (5.7) defines a generator of a HPD. The relevant role of spatial topology was first emphasized in [9] and treated in great detail in [16-18]. The degrees of freedom for class A globally homogeneous Bianchi models are also discussed in this reference; we will return to the question of degrees of freedom later.

Once we have completely eliminated the residual threediffeomorphism gauge invariance, the remaining gauge group is that of time reparametrizations. The lapse variable transforms, under the time reparametrization gauge group, as a scalar density,

$$
\delta_{\epsilon^{0}(t)} N=\epsilon^{0} \dot{N}+\dot{\epsilon}^{0} N
$$

and the three-metric variables $g_{a b}$ as a scalar:

$$
\delta_{\epsilon^{0}(t)} g_{a b}=\epsilon^{0} \dot{g}_{a b}
$$

\section{CONSEQUENCES OF $N^{a}=0$}

We have seen in Sec. III the non-commutativity between the operations of inserting the Killing conditions into the equations of motion or directly into the Lagrangian. Here we are going to consider another kind of non-commutativity, the one coming from plugging the gauge fixing $N^{a}=0$ into the Lagrangian. To make things clear, we only consider here the reduction of $\mathcal{L}$ in Eq. (3.3) to a reduced Lagrangian $\mathcal{L}_{\mathrm{GF}}$, prior to the introduction of the Killing conditions in it (GF stands for gauge fixing).

The important question is whether this new Lagrangian $\mathcal{L}_{\mathrm{GF}}$ is going to reproduce the same equations of motion derived from $\mathcal{L}$ under the gauge-fixing conditions $N^{a}=0$. The answer, from a general perspective which is summarized in Appendix C, can be found in [14], and in general is in the negative.

Bringing the results of [14] to our case, we find (here we use $[\mathcal{L}]$ for the Euler-Lagrange variation)

$$
[\mathcal{L}]=0, \quad N^{a}=0 \Leftrightarrow\left[\mathcal{L}_{\mathrm{GF}}\right]=0, \quad \mathcal{H}_{a}=0,
$$

where $\mathcal{H}_{a}$ are the Hamiltonian momentum constraints in the anholonomic basis and expressed in coordinate-velocity space. The $N^{a}$ have been set to zero in $\mathcal{H}_{a}$. If we also implement the Killing conditions in $\mathcal{H}_{a}$, we get

$$
\begin{aligned}
\mathcal{H}_{a} & =-|\omega| \frac{\sqrt{g}}{N}\left(\dot{g}_{b c \mid a}-\dot{g}_{a c \mid b}\right) g^{b c} \\
& =|\omega| \frac{\sqrt{g}}{N}\left(C_{a b}^{c} k_{c}^{b}+C_{b c}^{b} k_{a}^{c}\right),
\end{aligned}
$$

where $k_{c}^{b}=g^{b d} \dot{g}_{d c}$.

Therefore, if we use the completely reduced Lagrangian, with gauge fixing plus Killing conditions, with configuration variables $N, g_{a b}$, we must be aware that the correct equations of motion will require that initial conditions be taken such that

$$
C_{a b}^{c} k_{c}^{b}+C_{b c}^{b} k_{a}^{c}=0 .
$$

We show in Appendix $C$ that if the initial conditions satisfy Eq. (6.3), then Eq. (6.3) will hold at any time, provided the equations of motion are satisfied.

In class A models, where $C_{b c}^{b}=0$, Eq. (6.3) has only the term $C_{a b}^{c} k_{c}^{b}=0$. It is worth noting that, except for type I models, which have vanishing structure constants, in all other class A models, Eq. (6.3) must be enforced on the initial conditions. A diagonal form for the metric, for instance, will guarantee the fulfillment of Eq. (6.3) for most class A models as long as the structure constants are taken in the form displayed in [15] (the exception is the group of type $\mathrm{VI}_{-1}$ ). However, this diagonal form may not exhaust the possible physics available in these models, and we do not assume diagonality here.

Notice that the HPDs cannot be used to make the initial conditions satisfy Eq. (6.3): Under a change

$$
g_{a b} \rightarrow g_{a b}^{\prime}=M_{a}^{c} g_{c d} M_{b}^{d},
$$

the momentum constraints change as

$$
\mathcal{H}_{a}\left(\mathbf{g}^{\prime}\right)=(\operatorname{det} \mathbf{M}) M_{a}^{b} \mathcal{H}_{b}(\mathbf{g}) .
$$

The result of our analysis is the following: The completely reduced Lagrangian gives the correct equations of motion for class A models if and only if we tune the initial conditions (at $t=0) g_{a b}(0), \dot{g}_{a b}(0)$ in such a way that

$$
C_{a b}^{c} g^{b d}(0) \dot{g}_{d c}(0)=0 .
$$

To this result we must add the fact that two three-metrics, $g_{a b}$ and $g_{a b}^{\prime}$, are physically equivalent, that is, gauge related, if there is a HPD such that in the notation of Eq. (5.1) $g_{a b}^{\prime}=M_{a}^{c} g_{c d} M_{b}^{d}$. We must implement this fact in counting the number of independent initial conditions. In order to have a correct dynamics and a correct counting of the degrees of freedom for class A Bianchi models we must require that (1) the initial conditions must be chosen such that Eq. (6.4) is satisfied, and (2) initial conditions related by HPDs must be considered physically equivalent.

\section{HAMILTONIAN APPROACH}

The standard Hamiltonian Arnowitt-Deser-Misner (ADM) approach [15] is built upon the Lagrangian equation (3.3). Since $\mathcal{L}$ does not depend on $\dot{N}$ and $\dot{N}^{a}$, the conjugate variables $\mathcal{P}_{0}$ and $\mathcal{P}_{a}$ to $N$ and $N^{a}$ are the primary constraints in phase space. The canonical Hamiltonian has the form

$$
H=\int d^{3} x\left(N \mathcal{H}_{0}+N^{a} \mathcal{H}_{a}\right)
$$

The Dirac Hamiltonian $H_{\mathrm{D}}$ is formed by adding to $H$ a linear combination (with arbitrary functions $\lambda_{0}, \lambda_{a}$ ) of the primary constraints:

$$
H_{\mathrm{D}}=H+\int d^{3} x\left(\lambda^{0} \mathcal{P}_{0}+\lambda^{a} \mathcal{P}_{a}\right)
$$


Stability of the primary constraints under the evolution generated by $H_{\mathrm{D}}$ leads to constraints which are now secondary,

$$
\mathcal{H}_{0}=0, \quad \mathcal{H}_{a}=0,
$$

and no more constraints arise.

All eight constraints are first class, and the four independent gauge transformations that account for the diffeomorphism invariance are made out of them. For the sake of completeness let us write the gauge generators which act through the Poisson brackets in the whole phase space [19] (threespace integrations are understood for all repeated indices):

$$
G(t)=\mathcal{P}_{\mu} \dot{\xi}^{\mu}+\left(\mathcal{H}_{\mu}+N^{\rho} D_{\mu \rho}^{\nu} \mathcal{P}_{\nu}\right) \xi^{\mu},
$$

where the functions $D_{\mu \rho}^{\nu}$ describe the first class structure of the secondary constraints,

$$
\left\{\mathcal{H}_{\mu}, \mathcal{H}_{\rho}\right\}=D_{\mu \rho}^{\nu} \mathcal{H}_{\nu},
$$

and $\xi^{\mu}$ are arbitrary functions. The relationship of the transformations generated by $G(t)$ to the standard diffeomorphisms generated by a vector field $\epsilon^{\mu} \boldsymbol{\partial}_{\mu}$ is given by (see [19] for instance)

$$
\epsilon^{0}=\frac{\xi^{0}}{N}, \quad \epsilon^{a}=\xi^{a}-\frac{N^{a}}{N} \xi^{0} .
$$

On a given metric, every infinitesimal spacetime diffeomorphism $\epsilon^{\mu}$ is matched by $G(t)$ with the specific functions $\xi^{\mu}$ dictated by Eq. (7.6). This means that we have exactly the same gauge group either in the Lagrangian or in the Hamiltonian formalism, here extended to the phase space, and that in this last formalism the same steps must be taken to reduce the gauge group to the time-reparametrization invariance.

The implementation of the Killing conditions and the gauge fixing $N^{a}=0$ into the Hamiltonian equation (7.2) leads to the same conclusions we have arrived at in the Lagrangian formulation. In particular, only for class A models is the implementation of the Killing conditions into the Hamiltonian correct, in the sense that it gives the correct equations of motion. The reason is that the functional derivatives in the Hamiltonian formalism develop terms like the ones in Eq. (3.6). In fact, any of the $\mathcal{H}_{\mu}$ is of the form

$$
\mathcal{H}_{\mu}=|\omega| \widetilde{\mathcal{H}}_{\mu},
$$

in such a way that the three-space functional derivatives, corresponding to the Hamiltonian equations of motion, become (here X stands for $g_{a b}, \tilde{\pi}^{a b}$, where $\tilde{\pi}^{a b}$ is the canonical conjugate of $g_{a b}$ in the reduced formalism: $\left.\pi^{a b}=|\omega| \tilde{\pi}^{a b}\right)$

$$
\begin{aligned}
\frac{\delta \mathcal{H}_{\mu}}{\delta X}=|\omega| & {\left[\frac{\partial \widetilde{\mathcal{H}}_{\mu}}{\partial X}-\left(\mathbf{Y}_{a}+C_{a c}^{c}\right)\left(\frac{\partial \widetilde{\mathcal{H}}_{\mu}}{\partial \mathbf{Y}_{a} X}\right)\right.} \\
& \left.+\sum_{a \geqslant b}\left(\mathbf{Y}_{b}+C_{b d}^{d}\right)\left(\mathbf{Y}_{a}+C_{a c}^{c}\right)\left(\frac{\partial^{2} \widetilde{\mathcal{H}}_{\mu}}{\partial \mathbf{Y}_{a} \mathbf{Y}_{b} X}\right)\right] .
\end{aligned}
$$

(As a matter of fact, only $\mathcal{H}_{0}$ depends on second order space derivatives.)

To illustrate this last result, let us write the Poisson brackets for the momenta $\mathcal{H}_{a}$. They satisfy

$$
\begin{aligned}
&\left\{\int\right.\left.d^{3} x \mathcal{H}_{a} \xi_{1}^{a}, \int d^{3} x \mathcal{H}_{b} \xi_{2}^{b}\right\} \\
&=\int d^{3} x \mathcal{H}_{c}\left(\xi_{1}^{a} \xi_{2, a}^{c}-\xi_{2}^{a} \xi_{1, a}^{c}+C_{a b}^{c} \xi_{1}^{a} \xi_{2}^{b}\right)
\end{aligned}
$$

When we implement the Killing conditions on the quantity $\int d^{3} x \mathcal{H}_{a} \xi^{a}$, in order to get rid of the three-space dependence, it seems that we are bound to take the functions $\xi^{a}$ as constants (but this is not exactly true, as we will see shortly). This would generate diffeomorphisms of the type of Eq. (5.8), namely inner automorphisms for the left-invariant Lie algebra. Then $\widetilde{\mathcal{H}}_{a}$ reduces to

$$
\mathcal{H}_{a}^{\mathrm{red}}=C_{a b}^{c} \tilde{\pi}^{b d} g_{d c}+C_{b c}^{b} \tilde{\pi}^{c d} g_{d a}
$$

[this is the phase space version of Eq. (6.2)].

To continue, we must first introduce the Poisson brackets for the reduced theory. The reduced Poisson brackets $\{-,-\}_{R}$ are defined through a renormalization of the old Poisson brackets $\{-,-\}_{R}=V\{-,-\}$, where $V$ is the (perhaps infinite) right invariant volume element $V=\int \boldsymbol{\omega}^{1} \wedge \boldsymbol{\omega}^{2} \wedge \boldsymbol{\omega}^{3}=\int d^{3} x|\omega|$. Notice that as long as the Killing conditions hold we can invert the relation $\pi^{a b}=|\omega| \tilde{\pi}^{a b}$ to $\tilde{\pi}^{a b}=(1 / V) \int d^{3} x \pi^{a b}$. The commutation relations for $\mathcal{H}_{a}^{\text {red }}$ are

$$
\begin{aligned}
\left\{\mathcal{H}_{a}^{\mathrm{red}}, \mathcal{H}_{b}^{\mathrm{red}}\right\}_{R}= & C_{a b}^{c} \mathcal{H}_{c}^{\mathrm{red}}+C_{d c}^{d}\left(C_{a b}^{e} \tilde{\pi}^{c f} g_{f e}\right. \\
& \left.+C_{f a}^{f} \tilde{\pi}^{c e} g_{e b}-C_{f b}^{f} \tilde{\pi}^{c e} g_{e a}\right),
\end{aligned}
$$

whereas the reduction of the right side of Eq. (7.8) is, after factoring out the three-space volume,

$$
C_{a b}^{c} \mathcal{H}_{c}^{\text {red }} .
$$

It is only for class A models that we get this result in Eq. (7.10): Only for class A models do the implementation of the Killing conditions and the computation of the Poisson brackets commute.

\section{A. Homogeneity-preserving diffeomorphisms, revisited}

We have said, after Eq. (7.8), that the implementation of the Killing conditions on the generator $\int d^{3} x \mathcal{H}_{a} \xi^{a}$ does not require the functions $\xi^{a}$ to be constant. The reason is as follows. In the computations in Eq. (7.8) we have been dropping boundary terms. This is correct if our functions $\xi^{a}$ have a compact support on $\Sigma_{t}$. But we know from the Lagrangian analysis that some diffeomorphisms that do not vanish at the boundaries, for instance the HPDs, play an important role when we perform the dimensional reduction through the Killing conditions.

Consider the generator of three-space diffeomorphisms for the reduced set of variables $g_{a b}, \pi^{a b}$ : 


$$
\int d^{3} x \mathcal{H}_{a} \xi^{a}
$$

with

$$
\mathcal{H}_{a}=-|\omega| \sqrt{g}\left(|\omega|^{-1} \sqrt{g} \pi^{b c}\right)_{\mid b} g_{c a} .
$$

In order to get the standard result

$$
\delta g_{a b}=\left\{g_{a b}, \int d^{3} x \mathcal{H}_{a} \xi^{a}\right\}=\xi_{b \mid a}+\xi_{a \mid b},
$$

we always perform integration by parts in Eq. (7.11). Since the generator equation (7.11) acts locally, what we actually do in practice is to compute $\delta g_{a b}(t, x)$ using functions that are identical to $\xi_{a}$ in a neighborhood of a given point but that vanish at spatial infinity. If we perform directly the integration by parts in Eq. (7.11), we get

$$
G_{3}=\int d^{3} x \pi^{b c} \xi_{c \mid b}
$$

which differs from Eq. (7.11) by at most a boundary term.

Boundary terms may or may not exist depending on the topology of $\Sigma$. As long as gauge transformations on local functions are considered, Eq. (7.14) is as good as Eq. (7.11) to generate the transformations equation (7.13), but $G_{3}$ is better when we must work with arbitrary functions $\xi_{a}$ that are different from zero at the boundaries. Notice that whereas Eq. (7.11) vanishes on a solution of the equations of motion, because $\mathcal{H}_{a}$ is a constraint, Eq. (7.14) does not need to do so in the case when the functions $\xi_{a}$ do not vanish at the boundaries. Thus $G_{3}$ is not bound to be zero on a solution of the equations of motion. It is not a constraint, except when there are no boundaries in $\Sigma$ or for some other some particular cases that will be examined below.

The dimensional reductions of Eqs. (7.11) and (7.14) give different result, because integration by parts for the space coordinates does not exist in the reduced formalism. Now we apply the Killing conditions to Eq. (7.14):

$$
G_{3}=\int d^{3} x \pi^{b c} \xi_{c \mid b}=\int d^{3} x|\omega| \tilde{\pi}^{b c}\left(\mathbf{Y}_{b} \xi_{c}-\Gamma_{b c}^{d} \xi_{d}\right)
$$

where the connection coefficients $\Gamma_{b c}^{d}$ are

$$
\Gamma_{b c}^{d}=\frac{1}{2}\left(g_{c e} C_{b f}^{e} g^{f d}+g_{b e} C_{c f}^{e} g^{f d}-C_{b c}^{d}\right)
$$

(here $g^{f d}=e^{f d}$ since $N^{a}=0$ ). Therefore,

$$
G_{3}=\int d^{3} x|\omega| \tilde{\pi}^{b c} g_{c d}\left(\mathbf{Y}_{b} \xi^{d}-C_{b e}^{d} \xi^{e}\right)
$$

Dimensional reduction requires us to factor out the volume $V=\int d^{3} x|\omega|$. This means that we need the remaining piece in the integrand, $\tilde{\pi}^{b c} g_{c d}\left(\mathbf{Y}_{b} \xi^{d}-C_{b e}^{d} \xi^{e}\right)$, to be independent of the space coordinates. Since $\tilde{\pi}^{b c}$ and $g_{c d}$ are already space independent because they satisfy the Killing conditions, we end up with the requirement

$$
\mathbf{Y}_{b} \xi^{d}-C_{b e}^{d} \xi^{e}=B_{b}^{d}
$$

with $B_{b}^{d}$ a constant matrix. This condition is exactly Eq. (5.2), the condition for HPDs, expressed in the dual basis. Thus we see that the dimensional reduction in phase space has room for and only for the HPDs we already found in the Lagrangian formalism. Then $G_{3}$ becomes $G_{3}=V B_{b}^{d} \tilde{\pi}^{b c} g_{c d}$, and since the reduced Poisson brackets are $\{-,-\}_{R}=V\{-,-\}$, we get, for the canonical generator of HPDs in the reduced formalism,

$$
\widetilde{G}_{3}=B_{b}^{d} \widetilde{\pi}^{b c} g_{c d} \text {. }
$$

We find here another special feature of the Bianchi models, for the $\widetilde{G}_{3}$ in Eq. (7.17) generates gauge transformations, HPDs, and yet it is not a constraint of the reduced formalism by itself, except for the case in class $A$ models of inner automorphisms. In this last case, we have $B_{b}^{d}=B^{c} C_{c b}^{d}$, and $\widetilde{G}_{3}$ for class A models becomes $\widetilde{G}_{3}=B^{a} \mathcal{H}_{a}^{\text {red }}$, with $\mathcal{H}_{a}^{\text {red }}$ given in Eq. (7.9) with $C_{b c}^{b}=0$. These results completely match the ones previously obtained in the Lagrangian analysis.

The generators of HPDs that define automorphisms of the Lie algebra that are not inner automorphisms (called "outer', HPDs in [9]) are always constants of motion [9] of the reduced formalism. They are enforced to be constraints if $\Sigma$ has no boundaries, although these constraints cannot be retrieved as such constraints from the reduced formalism by itself. In any case, either being constants of motion or constraints, they always generate gauge transformations in the reduced formalism. These transformations, in either the Lagrangian or the Hamiltonian picture, are available as gauge transformations from the outset, as a consequence of the rationale of the reduction procedure, but they cannot be retrieved as gauge transformations from the reduced formalism by itself.

A comment is in order for class B models. The generators, Eq. (7.17), give the HPDs for class B as well as class A models. In particular, $C_{a b}^{d} \tilde{\pi}^{b c} g_{c d}$ generates the inner automorphisms associated, in the configuration space picture, with the right-invariant vector $\mathbf{Y}_{a}$. Nevertheless, $C_{a b}^{d} \tilde{\pi}^{b c} g_{c d}$ is not necessarily a constraint for the class B models because the true momentum constraints are those of Eq. (7.9), as one can directly verify from the equations of motion. Oddly enough, in class B models at least one of the generators of the residual gauge transformations associated with inner automorphisms is not a constraint. Also, according to Eq. (7.10), these constraints, two of them, are no longer first class. These results hint that the Lagrangian or Hamiltonian formulation for class B models is not possible in general.

Notice that in the case of three-manifolds $\Sigma$ with no boundaries, the possible new constraints $\widetilde{G}_{3}$ in Eq. (7.17) appear also in the Lagrangian formalism by simply writing down the process from Eq. (7.11) to Eq. (7.14) in configuration-velocity variables.

One could think that the mechanism of integration by parts, as used to transform Eq. (7.11) into Eq. (7.14), and that 
helped us find the whole set of HPDs in phase space, could perhaps be used in class B models to transform the reduced $\mathcal{H}_{0}$ into the correct generator of time translations. In fact, some unwanted pieces can be eliminated this way, but this does not solve the problem.

\section{B. Degrees of freedom}

Notice also that the tangency of the Hamiltonian evolution operator equation (7.1) to the gauge fixing surface defined by $N^{a}=0$ implies $\lambda^{a}=0$. On the other hand, once the gauge fixing $N^{a}=0$ has been introduced into $H_{\mathrm{D}}$, the stability of the primary constraints $\mathcal{P}_{a}=0$ will no longer produce the secondary constraints $\mathcal{H}_{a}=0$. This result parallels that of Eq. (6.1). As a matter of fact, and since they have become second class constraints, these three couples of canonical variables $N^{a}, \mathcal{P}_{b}$ are readily eliminated from the formalism by taking the Dirac brackets, which are nothing but the ordinary Poisson brackets for the rest of the variables (the same number of variables, $N^{a}, \dot{N}^{b}$, are eliminated at the same stage in the Lagrangian formulation). The important point is that the momentum constraints are gone from the reduced formalism, but yet they must be implemented (in case they do not vanish identically) from the outset as restrictions imposed on the initial conditions, if we want to have the right equations of motion.

It is remarkable also that the generators of HPDs in the dimensionally reduced theory are not necessarily constraints, except for the case of inner automorphisms and class A models. We still need to fix all the residual gauge freedom corresponding to the three-diffeomorphisms, because these three-diffeomorphisms have been with us since the beginning. We are in the same situation as we were in the Lagrangian formulation, and therefore the same HPDs, exactly the same, appear here, as we have just shown. That is why the degrees of freedom in both the Lagrangian and the Hamiltonian formalisms coincide.

In [9] a discrepancy between Lagrangian and Hamiltonian degrees of freedom is argued on the basis that the only HPDs available in Hamiltonian formalism are those yielding inner automorphisms. Our analysis differs from the one in [9] in that we show that the residual three-diffeomorphism invariance that still needs to be fixed corresponds to the HPDs that we found earlier in the Lagrangian formalism. And this is something that one knows in advance through the process of reducing the original gauge group.

The claims in [9] are opposed in [20], where it is argued that the degrees of freedom for both the Lagrangian and Hamiltonian formalisms are the same. However, they base their claim on rejecting the HPDs that are not associated with inner automorphisms as residual gauge transformations that need to be fixed. In the next section we show that all the HPDs are indeed residual gauge transformations that describe redundancy in the initial conditions of the system and therefore do need to be fixed.

\section{CONCLUSIONS}

We have proved that class A Bianchi models allow for a Lagrangian formulation as a mechanical particle-like system (finite number of degrees of freedom, in contrast with field theory) as long as the setting of the initial conditions is taken in a certain way.

In our transit toward this result we have proceeded as follows: We have carefully produced a partial gauge fixing in four steps, to reduce the initial gauge group generated by the four-diffeomorphism invariance to time reparametrization invariance. As a consequence, the troubles that beset class B Bianchi models are identified as obstructions to the commutativity between two processes: implementing the Killing conditions either into the equations of motion or directly into the Lagrangian, followed by deriving the equations of motion.

We distinguish, as regards the Lagrangian or Hamiltonian formulations, two types of problems, which correspond, respectively, to the implementation of the Killing conditions into the Lagrangian and to the implementation of the gauge fixing that sets the shift variables to zero. As we have just said, the first problem prevents the class B Bianchi models from having a reduced Lagrangian or Hamiltonian formulation in general. The second tells us that the initial conditions must be chosen to satisfy some former constraints (the momentum constraints) of the original theory, even though they are no longer constraints for the reduced one.

We have shown that the Hamiltonian formalism has the same gauge freedom that is available in the Lagrangian formulation. The reduction of the gauge group in phase space follows exactly the same steps as in configuration-velocity space, and the same features, the same problems, the same considerations, and the same results apply as well. The fact that the reduced momenta $\mathcal{H}_{a}$ in class A models can only generate HPDs that are inner automorphisms of the Lie algebra has its counterpart in configuration-velocity space in that these HPDs are the only ones that can be derived as gauge transformations from the reduced Lagrangian on its own. But that does not mean that the HPDs associated with the "outer" automorphisms, if there are any, do not need to be quotiented out. Rather, all HPDs must be quotiented out, either in the Hamiltonian or in the Lagrangian formalism.

Also, what is and what is not a gauge symmetry and the role of the residual gauge symmetries have been analyzed in detail. We think that our considerations throw a definitive light on some issues that have not yet been settled in the literature $[9,20]$.

Through our analysis, the gauge group is reduced in several stages, and residual gauge symmetries appear. According to the fact that they all proceed from the original gauge group, we claim that these residual gauge symmetries must be fixed thoroughly. We think that this is an important point that deserves further elaboration below.

Let us show the need to fix the gauge for the residual gauge group of three-space diffeomorphisms that appears at the end of the third step of the gauge fixing procedure (Sec. IV). Consider that we have a solution $g_{i j}(x, t), N(t)$ of the Einstein equations with $N^{i}=0$ and with initial conditions at $t=0$. Now consider the action of the infinitesimal diffeomorphism transformation defined by the vector field $\epsilon^{i} \boldsymbol{\partial}_{i}$,

$$
\epsilon^{i}(x, t)=f(t) \tilde{\epsilon}^{i}(x),
$$


where $f$ has been chosen to satisfy

$$
f(0)=0, \quad \dot{f}(0)=; \quad f(t)=1, \quad \dot{f}(t)=0 \quad \text { for } t \geqslant 1 .
$$

Since this vector field generates a foliation-preserving diffeomorphism, it will define a gauge transformation which is still allowed in the formalism after the first-step gauge fixing has been done. The transformed metric $g_{i j}^{\prime}(x), N^{\prime}(x), N^{\prime i}(x)$ is also a solution of the Einstein equations. Notice that both metrics $g_{\mu \nu}$ and $g_{\mu \nu}^{\prime}$ share the same initial conditions at $t$ $=0$. It is then obvious that the correct interpretation of a gauge transformation dictates that $g_{\mu \nu}$ and $g_{\mu \nu}^{\prime}$ be physically equivalent and must be so for any other time we take for the setting of the initial conditions.

Now consider the relation between these two metrics but with initial conditions taken at $t=1$. They are related by an infinitesimal three-space diffeomorphism generated by the time-independent vector field $\tilde{\epsilon}^{i}(x) \boldsymbol{\partial}_{i}$. The two metrics satisfy the gauge $N^{i}=0$. The gauge transformation that connects them is not localizable in time (being different from the identity only within a finite time interval), which is the point made in [20] to deem this transformation as non-gauge, and yet both metrics must be physically identified. This result proves that we must fix the gauge even for the three-space diffeomorphisms, namely, the residual gauge group. It is in this place that the HPDs play the significant role we have seen in Secs. V and VII.

Our analysis agrees with that of [9] with regard to the Lagrangian formulation, and we agree with their spacetime counting of the degrees of freedom (see the table in [9]; also see [16-18] for a counting of degrees of freedom in models with compactified spatial sections). But we differ in other respects. Let us make the differences clear; they concern the status of the HPDs associated with "outer' automorphisms of the right invariant Lie algebra.

In [9], “outer' HPDs, that is, HPDs yielding automorphisms of the Lie algebra that are not inner automorphisms, count as gauge degrees of freedom in the Lagrangian formulation, but are considered non-gauge symmetries in phase space. In [20], these "'outer' HPDs are always taken in both formulations as non-gauge symmetries. Instead, from our systematic procedure of reducing the gauge group, we deduce that the "outer" HPDs are gauge transformations in both the Lagrangian and the Hamiltonian formalisms, and they always count as gauge degrees of freedom.

Also see our comments in Appendix D. For example, we explicitly consider the case of the Bianchi type I model, where the number of degrees of freedom is 1 (if the surfaces of homogeneity have the topology of $\mathbb{R}^{3}$ ). It may appear to some people that an odd number of degrees of freedom in a Hamiltonian formalism is somehow not correct, but that is the case here, and we discuss this matter in a bit more detail in Appendix D.

Summing up, we have exhibited in a simple way the two different problems that appear when we implement the Killing conditions and gauge fixing into the Lagrangian or into the Hamiltonian. The first problem prevents the class $\mathrm{B} \mathrm{Bi}$ anchi models from having Lagrangian or Hamiltonian formulations (except possibly in special cases), whereas the sec- ond is solved by introducing some requirements on the initial conditions. We have also shown that there is no ambiguity or matter of interpretation in what must be understood as a gauge transformation, either in the Lagrangian or the Hamiltonian formalism. Finally our analysis proves that the number of degrees of freedom in both formalisms is always the same.

\section{ACKNOWLEDGMENTS}

J.M.P. would like to thank the Center for Relativity of The University of Texas at Austin for its hospitality. J.M.P. acknowledges support by CICYT contracts AEN95-0590 and GRQ 93-1047 and wishes to thank the Comissionat per a Universitats i Recerca de la Generalitat de Catalunya for a grant.

\section{APPENDIX A: GENERAL SPATIALLY HOMOGENEOUS METRIC}

In order to demonstrate some of our ideas explicitly, we give here formulas for the connection, curvature, and field equations for a general spatially homogeneous metric, including a general lapse function and shift vector. A word on the calculational procedure: It is easiest to use an orthonormal basis adapted to the Killing structure,

$$
\mathbf{g}=\eta_{\mu \nu} \boldsymbol{\sigma}^{\mu} \boldsymbol{\sigma}^{n u}=-\left(\boldsymbol{\sigma}^{0}\right)^{2}+\delta_{i j} \boldsymbol{\sigma}^{i} \boldsymbol{\sigma}^{j}
$$

where the basis is defined by

$$
\boldsymbol{\sigma}^{0}=N \mathbf{d} t, \quad \boldsymbol{\sigma}^{i}=b_{a}^{i}\left(\boldsymbol{\omega}^{a}+N^{a} \mathbf{d} t\right)
$$

where $N, N^{a}, g_{a b}$ are functions only of $t$, and we use the convention that boldface denotes a tensor or a form; the oneforms $\boldsymbol{\omega}^{a}$ obey the following relation, indicating that they are invariant under the isometry group whose Lie algebra is defined by the structure constants $C_{b c}^{a}$ :

$$
\mathbf{d} \boldsymbol{\omega}^{a}=\frac{1}{2} C_{b c}^{a} \boldsymbol{\omega}^{b} \wedge \boldsymbol{\omega}^{c}
$$

Note that the three-metric is defined by

$$
g_{a b}=\delta_{i j} b_{a}^{i} b_{b}^{j}
$$

and we will consistently use $i, j, \ldots$ for orthonormal indices, and $a, b, c, \ldots$ for ordinary three-indices. The matrix $\mathbf{B}=\left(b_{a}^{i}(t)\right)$ represents an arbitrary square root of the metric; precisely what form it takes will be irrelevant. The inverse of the three-metric is defined by

$$
e^{a c} g_{c b}=\delta_{b}^{a}
$$

in order not to confuse it with the $a b$ components of the contravariant four-metric. The inverse of $\mathbf{B}$ is the matrix $\mathbf{A}=\left(a_{i}^{a}\right)$, so that

$$
e^{a b}=a_{i}^{a} a_{j}^{b} \delta^{i j} \quad \text { where } a_{j}^{a} b_{a}^{i}=\delta_{j}^{i} \Leftrightarrow a_{i}^{a} b_{b}^{i}=\delta_{b}^{a} .
$$


The first Cartan equations with the torsion set equal to zero are

$$
\mathbf{d} \boldsymbol{\sigma}^{\mu}=-\boldsymbol{\sigma}^{\sigma} \wedge \boldsymbol{\sigma}_{\sigma}^{\mu}
$$

where $\boldsymbol{\sigma}_{\nu}^{\mu}$ are the connection one-forms, used in forming the covariant derivative of a tensor. The metric compatibility equations (covariant derivative of the metric equals zero), when the metric components are constants, as in an orthonormal basis, here read

$$
\eta_{\mu \sigma} \boldsymbol{\sigma}_{\nu}^{\sigma}=-\eta_{\nu \sigma} \boldsymbol{\sigma}_{\mu}^{\sigma}
$$

Equations (A7) and (A8) uniquely determine the connection one-forms. The second Cartan equations are

$$
\frac{1}{2} R_{\nu \sigma \tau}^{\mu} \boldsymbol{\sigma}^{\sigma} \wedge \boldsymbol{\sigma}^{\tau}=\mathbf{d} \boldsymbol{\sigma}_{\nu}^{\mu}+\boldsymbol{\sigma}_{\sigma}^{\mu} \wedge \boldsymbol{\sigma}_{\nu}^{\sigma}
$$

they determine the Riemann tensor components (see [15] for more details).

It is convenient to define a matrix related to the logarithmic derivative of $\mathbf{B}$ and its symmetric and antisymmetric parts as the matrices $\mathbf{K}, \mathbf{L}, \mathbf{M}$. It is also convenient to define the orthonormal projection of the structure constants (which then become a time-dependent array $\mathbf{D}$ ) plus another array $\mathbf{E}$ by (here an overdot denotes $d / d t$ )

$$
\begin{gathered}
K_{i j}=\frac{1}{N}\left(\dot{b}_{a}^{i} a_{j}^{a}-b_{a}^{i} N^{b} a_{j}^{c} C_{b c}^{a}\right), \\
L_{i j}=\frac{1}{2}\left(K_{i j}+K_{j i}\right), \quad M_{i j}=\frac{1}{2}\left(K_{i j}-K_{j i}\right), \\
D_{j k}^{i}=b_{a}^{i} a_{j}^{b} a_{k}^{c} C_{b c}^{a}, \quad E_{i j k}=D_{j k}^{i}-D_{i k}^{j}-D_{i j}^{k} .
\end{gathered}
$$

With these definitions, we have, for the curls of the orthonormal basis forms,

$$
\mathbf{d} \boldsymbol{\sigma}^{0}=0, \quad \mathbf{d} \boldsymbol{\sigma}^{i}=K_{i j} \boldsymbol{\sigma}^{0} \wedge \boldsymbol{\sigma}^{j}+\frac{1}{2} D_{j k}^{i} \boldsymbol{\sigma}^{j} \wedge \boldsymbol{\sigma}^{k}
$$

The connections forms are

$$
\boldsymbol{\sigma}_{i}^{0}=\boldsymbol{\sigma}_{0}^{i}=L_{i j} \boldsymbol{\sigma}^{j}, \quad \boldsymbol{\sigma}_{j}^{i}=-M_{i j} \boldsymbol{\sigma}^{0}+\frac{1}{2} E_{i j k} \boldsymbol{\sigma}^{k}
$$

The results for the independent components of the Riemann tensor are most conveniently displayed after raising an index (using $\delta^{i j}$ ):

$$
\begin{aligned}
& R_{0 j}^{0 i}=\frac{1}{N} \dot{L}_{i j}+L_{i k} L_{j k}-L_{i k} M_{j k}-L_{j k} M_{i k}, \\
& R_{k l}^{0 i}=L_{i j} D_{k l}^{j}+\frac{1}{2}\left(L_{j k} E_{j i l}-L_{j l} E_{j i k}\right), \\
& R_{k l}^{i j}=L_{i k} L_{j l}-L_{i l} L_{j k}
\end{aligned}
$$

$$
-\frac{1}{4} E_{i m k} E_{j m l}+\frac{1}{4} E_{i m l} E_{j m k}+\frac{1}{2} E_{i j m} D_{k l}^{m} .
$$

The Ricci tensor components, the scalar curvature, and the Einstein tensor components are defined by

$$
R_{\nu}^{\mu}=R_{\nu \sigma}^{\mu \sigma}, \quad R=R_{\sigma}^{\sigma}, \quad G_{\nu}^{\mu}=R_{\nu}^{\mu}-\frac{1}{2} R \delta_{\nu}^{\mu} .
$$

The evolution equations (in vacuum) are setting the spacespace components of the Ricci tensor to zero, where

$$
\begin{aligned}
R_{j}^{i}= & \frac{1}{N} \dot{L}_{i j}-L_{i k} M_{j k}-L_{j k} M_{i k}+L_{k k} L_{i j}+\frac{1}{2}\left(D_{j k}^{i}+D_{i k}^{j}\right) D_{k l}^{l} \\
& -\frac{1}{2} D_{i k}^{l}\left(D_{j l}^{k}+D_{j k}^{l}\right)+\frac{1}{4} D_{k l}^{i} D_{k l}^{j} .
\end{aligned}
$$

Next we display the time-time and time-space components of the Einstein tensor; these set to zero are the constraint equations on initial value data:

$G_{0}^{0}=\frac{1}{2} L_{s t} L_{s t}-\frac{1}{2}\left(L_{s s}\right)^{2}+\frac{1}{2} D_{s t}^{t} D_{s u}^{u}+\frac{1}{4} D_{s u}^{t} D_{s t}^{u}+\frac{1}{8} D_{t u}^{s} D_{t u}^{s}$

$G_{i}^{0}=-L_{i j} D_{j k}^{k}-L_{j k} D_{k i}^{j}$

Finally, we will need the scalar curvature in order to display the reduced Lagrangian. In the action integral the volume element is really

$$
\boldsymbol{\sigma}^{0} \wedge \boldsymbol{\sigma}^{1} \wedge \boldsymbol{\sigma}^{2} \wedge \boldsymbol{\sigma}^{3}=N \sqrt{g} \mathbf{d} t \wedge \boldsymbol{\omega}^{1} \wedge \boldsymbol{\omega}^{2} \wedge \boldsymbol{\omega}^{3}
$$

where $g=\operatorname{det}\left(g_{a b}\right)$, and the spatial integral can be set to the constant $V$ :

$$
\iiint \omega^{1} \wedge \omega^{2} \wedge \omega^{3}=V
$$

The action integral is therefore

$$
\mathcal{I}=\int \mathcal{L} V d t
$$

where

$$
\begin{aligned}
\mathcal{L}= & R N \sqrt{g} \\
= & \left(2 \sqrt{g} L_{s s}\right) \cdot-2 \sqrt{g} N^{a} b_{a}^{s} D_{s u}^{u} L_{s s}+N \sqrt{g}\left(L_{s t} L_{s t}-\left(L_{s s}\right)^{2}\right. \\
& \left.-D_{s t}^{t} D_{s u}^{u}-\frac{1}{2} D_{s u}^{t} D_{s t}^{u}-\frac{1}{4} D_{t u}^{s} D_{t u}^{s}\right)
\end{aligned}
$$

and note that we have separated out a total time derivative. Here it is best to be explicit, and we display the Lagrangian in terms of the configuration space variables $N, N^{a}, g_{a b}$ : 


$$
\begin{aligned}
\mathcal{L}= & \frac{\sqrt{g}}{N}\left[-\frac{1}{4}\left(e^{a b} \dot{g}_{a b}\right)^{2}+\frac{1}{4} \dot{g}_{a b} \dot{g}_{c d} e^{a c} e^{b d}-\dot{g}_{a b} e^{b c} N^{d} C_{d c}^{a}\right. \\
& \left.+\left(N^{a} C_{a b}^{b}\right)^{2}+\frac{1}{2} g_{a b} e^{c d} N^{e} N^{f} C_{e c}^{a} C_{f d}^{b}+\frac{1}{2} N^{e} N^{f} C_{e b}^{a} C_{f a}^{b}\right] \\
& -N \sqrt{g}\left[+e^{b c} C_{b a}^{a} C_{c d}^{d}+\frac{1}{2} e^{c d} C_{c b}^{a} C_{d a}^{b}\right. \\
& \left.+\frac{1}{4} g_{a d} e^{b e} e^{c f} C_{b c}^{a} C_{e f}^{d}\right] .
\end{aligned}
$$

The vacuum field equations are most conveniently written as four constraint equations and six evolution equations:

$$
G_{0}^{0}=0, \quad G_{i}^{0}=0, \quad R_{j}^{i}=0 .
$$

These equations can be derived from the Einstein-Hilbert Lagrangian density before imposing any symmetry requirements and then imposing the symmetry requirements after the equations have been derived. We will compare these equations with those derived from the reduced Lagrangian $\mathcal{L}$, in other words, by imposing the symmetry requirements first and then deriving equations.

The equation $\delta \mathcal{L} / \delta N=0$ is readily seen to be exactly $G_{0}^{0}=0$ (up to a factor of $2 \sqrt{g}$ ).

The equations $\delta \mathcal{L} / \delta N^{a}=0$ (after canceling $N$ and $\sqrt{g}$ ) are

$$
\begin{aligned}
0= & +\dot{g}_{d b} g^{b c} C_{c a}^{d}+2 C_{a c}^{c} N^{b} C_{b d}^{d} \\
& +g_{e f} g^{c d} N^{b} C_{b d}^{f} C_{a c}^{e}+N^{b} C_{b c}^{d} C_{a d}^{c} .
\end{aligned}
$$

This is not proportional to the $G_{i}^{0}$ equation in a class B model; the difference (up to nonzero factors) is

$$
\left[\dot{g}_{a b} g^{b f}+2 C_{a c}^{c} N^{f}-g_{a e} g^{c f} N^{b} C_{b c}^{e}\right] C_{f d}^{d} .
$$

In other words, if the lapse is kept in, then all of the constraint equations can be derived for the class A models. In class $\mathrm{B}$, if these terms happen to be zero anyway, well and good, but they will not automatically vanish. Note several things about the terms in Eq. (A23): The first one is independent of $N^{a}$; therefore, one might hope that adding an appropriate term to the Lagrangian might remove it (a term, that is, linear in $N^{a}$ ). The second term has $N^{a}$ multiplied by a matrix which is symmetric; supposedly it too could be removed with an appropriate term in the Lagrangian. The last term, however, has $N^{a}$ times a matrix which is not symmetric; it would seem that there is no hope of generating this term by adding something to the Lagrangian.

\section{APPENDIX B: SPATIALLY HOMOGENEOUS MAXWELL THEORY}

A relatively simple system which illustrates many of our ideas is that of a spatially homogeneous electromagnetic potential which obeys the Maxwell equations. (Here we start with the four-vector potential rather than with a spatially homogeneous field tensor, which of course could be produced using a non-homogeneous potential vector.) The back- ground metric is taken to be a simple spatially homogeneous one, and so we do not require that it obey any particular field equations. We take the metric to have components $\eta_{\mu \nu}$ in an invariant basis:

$$
\mathbf{g}=-\mathbf{d} t^{2}+\delta_{i j} \boldsymbol{\omega}^{i} \boldsymbol{\omega}^{j}
$$

where

$$
\mathbf{d} \boldsymbol{\omega}^{i}=\frac{1}{2} C_{j k}^{i} \boldsymbol{\omega}^{j} \wedge \boldsymbol{\omega}^{k} .
$$

Note that there is a freedom to transform the spatial basis $\left\{\boldsymbol{\omega}^{i}\right\}$ by an orthogonal transformation but not necessarily by a general linear transformation.

The vector potential actually is an equivalence class of one-forms related by gauge transformations. Here we demand that the class contain at least one member which is spatially homogeneous. If that one is expressed in the invariant basis, its components are functions only of $t$,

$$
\mathbf{A}=A_{0}(t) \mathbf{d} t+A_{i}(t) \boldsymbol{\omega}^{i},
$$

and it produces the following field 2-form:

$$
\mathbf{F}=\mathbf{d} \mathbf{A}=\dot{A}_{i} \mathbf{d} t \wedge \boldsymbol{\omega}^{i}+\frac{1}{2} A_{i} C_{j k}^{i} \boldsymbol{\omega}^{j} \wedge \boldsymbol{\omega}^{k} .
$$

Note that $A_{0}$ has disappeared; it plays no part either in the Maxwell equations or in the Lagrangian. It can be made to vanish by a gauge transformation of the kind $\mathbf{A} \rightarrow \mathbf{A}^{\prime}=\mathbf{A}$ $+\mathbf{d} \lambda(t)$.

We choose to concentrate on the metric as expressed in an invariant basis; this is a choice of metric gauge. Maxwell theory cannot be expressed without at least some reference to a background spacetime metric, and so this metric gauge affects the electromagnetic potential. We also choose to concentrate on the vector potential within its equivalence class which explicitly has a vanishing Lie derivative with respect to the generators of the invariance group. This, too, is a choice of gauge, though, as we discuss below, in some cases there is still some residual gauge freedom.

The easiest way to calculate the Maxwell equations (in vacuum) is first to write down the dual field 2-form:

$$
{ }^{*} \mathbf{F}=\frac{1}{2} \epsilon_{i j k} A_{s} C_{j k}^{s} \mathbf{d} t \wedge \boldsymbol{\omega}^{i}-\frac{1}{2} \epsilon_{i j s} \dot{A}_{s} \boldsymbol{\omega}^{i} \wedge \boldsymbol{\omega}^{j},
$$

where $\epsilon_{i j k}$ is the Levi-Cività symbol, equal to \pm 1 if ( $\left.i j k\right)$ is an even or odd permutation of (123) and to zero otherwise. The Maxwell equations are

$$
\begin{aligned}
0= & \mathbf{d}^{*} \mathbf{F} \\
= & -\frac{1}{2}\left(\boldsymbol{\epsilon}_{s t u} \ddot{A}_{s}+\frac{1}{2} \epsilon_{i j k} A_{s} C_{j k}^{s} C_{t u}^{i}\right) \mathbf{d} \mathbf{t} \wedge \boldsymbol{\omega}^{t} \wedge \boldsymbol{\omega}^{u} \\
& -\frac{1}{2} \epsilon_{i s k} \dot{A}_{k} C_{t u}^{i} \boldsymbol{\omega}^{s} \wedge \boldsymbol{\omega}^{t} \wedge \boldsymbol{\omega}^{u},
\end{aligned}
$$

and they are equivalently written as 


$$
\begin{gathered}
0=\dot{A}_{j} C_{j s}^{s}, \\
0=\ddot{A}_{i}+\frac{1}{2} A_{s} C_{j k}^{s} C_{j k}^{i}+A_{s} C_{i t}^{s} C_{t u}^{u} .
\end{gathered}
$$

One can double-check these equations by forming the connection one-forms and computing $F_{; \sigma}^{\mu \sigma}=0$.

The action integral for a general Maxwell vector potential is $\left({ }^{4} g\right.$ is the determinant of the spacetime metric in a coordinate system)

$$
\mathcal{I}=\int \frac{1}{4} F^{\mu \nu} F_{\mu \nu} \sqrt{\left|{ }^{4} g\right|} d^{4} x .
$$

In this case, $\sqrt{\left|{ }^{4} g\right|}$ is independent of $t$, and so the reduced Lagrangian $\mathcal{L}_{R}$ is

$$
\mathcal{L}_{R}=\frac{1}{4} F^{\sigma \tau} F_{\sigma \tau}=-\frac{1}{2} \dot{A}_{s} \dot{A}_{s}+\frac{1}{4} A_{s} A_{t} C_{j k}^{s} C_{j k}^{t} .
$$

The Euler-Lagrange equations $0=\delta \mathcal{L}_{R} / \delta A_{i}$ are

$$
0=\ddot{A}_{i}+\frac{1}{2} A_{s} C_{j k}^{i} C_{j k}^{s} .
$$

It is clear that in class B models Eqs. (B7) and (B10) do not agree. There are two differences: Equation (B7a) is a constraint equation, and it simply cannot come from a variational principle which is homogeneous quadratic in the velocities. Equation (B7b) has the term $A_{s} C_{i t}^{s} C_{t u}^{u}$ which does not appear in Eq. (B10). Notice that Eq. (B7a) is automatically satisfied in the class A case $\left(C_{j s}^{s}=0\right)$, and also Eqs. (B7b) and (B10) then do agree.

One thing which is true, however, is that the constraint equation (B7a) is compatible both with Eqs. (B7b) and (B10). To see this fact, take the time derivative of Eq. (B7a):

$$
0=\ddot{A}_{j} C_{j s}^{s} .
$$

When Eq. (B7b) is multiplied by $C_{i s}^{s}$, the result is

$$
0=C_{i s}^{s} \ddot{A}_{i}+\frac{1}{2} A_{t} C_{j k}^{t} C_{i s}^{s}+A_{j} C_{i t}^{j} C_{t u}^{u} C_{i s}^{s}
$$

The middle term vanishes because of the Jacobi identity, which in a three-dimensional Lie algebra is equivalent to $0=C_{i j}^{s} C_{s t}^{t}$. The last term, which would be absent anyway if Eq. (B10) had been used, vanishes as a consequence of the antisymmetry of $C_{i t}^{j}$ in its lower indices.

Thus, even if the constraint equation (B7a) were put in by hand, the problem would remain whether any variational principle could reproduce the last term in Eq. (B7b).

We now turn to gauge transformations. A gauge transformation here is the addition to the vector potential of a homogeneous one-form whose curl vanishes (and therefore which can at least locally be expressed as the curl of a function). Let this one-form be

$$
\boldsymbol{\kappa}=\kappa_{0} \mathbf{d} t+\kappa_{i} \boldsymbol{\omega}^{i}
$$

where the components $\kappa_{\mu}$ are functions only of $t$. We require that the curl of $\boldsymbol{\kappa}$ be zero:

$$
0=\mathbf{d} \boldsymbol{\kappa}=\dot{\kappa}_{i} \mathbf{d} t \wedge \boldsymbol{\omega}^{i}+\frac{1}{2} \kappa_{i} C_{j k}^{i} \boldsymbol{\omega}^{j} \wedge \boldsymbol{\omega}^{k}
$$

Therefore $\kappa_{0}$ is arbitrary, and $\kappa_{i}$ must be a set of constants subject to the condition

$$
\kappa_{i} C_{j k}^{i}=0 .
$$

Only Bianchi types VIII and IX require that $\kappa_{i}$ be zero. In fact, in a class B model, $\kappa_{i}$ may be taken to be proportional to $C_{i j}^{j}$.

We illustrate with the example of the general class B model, in which only $C_{1 s}^{s} \neq 0$ (this prescription can always be satisfied in any class B model by using an orthogonal transformation of the invariant basis). The Jacobi identity then requires that $C_{i j}^{1}=0$. The reduced Lagrangian in this case is

$$
\mathcal{L}_{R}=-\frac{1}{2}\left(\dot{A}_{1}^{2}+\dot{A}_{2}^{2}+\dot{A}_{3}^{2}\right)+\frac{1}{4} A_{A} A_{B} C_{j k}^{A} C_{j k}^{B},
$$

where the indices $A, B$ range only over $(2,3)$. The EulerLagrange equations are

$$
0=\ddot{A}_{1}, \quad 0=\ddot{A}_{A}+\frac{1}{2} A_{B} M_{A B},
$$

where

$$
M_{A B} \equiv C_{j k}^{A} C_{j k}^{B}
$$

The Maxwell equations (B7) read

$$
\begin{gathered}
0=\dot{A}_{1}, \\
0=\ddot{A}_{1}, \quad 0=\ddot{A}_{A}+\frac{1}{2} A_{B} M_{A B}+A_{B} N_{A B},
\end{gathered}
$$

where

$$
N_{A B} \equiv C_{A 1}^{B} C_{1 s}^{s} \text {. }
$$

The reduced Maxwell equations thus differ in two ways from the Euler-Lagrange equations: First, the Maxwell equations have the constraint equation $\dot{A}_{1}=0$. The residual gauge freedom allowed by Eq. (B13) says that $A_{1}$ can be made zero by a choice of gauge. In contrast, the Euler-Lagrange equations only require that $A_{1}$ be at most a linear function of $t$, and the gauge freedom only will allow the value of $A_{1}$ to be set to zero at a particular time. It is not, however, difficult to add the constraint $\dot{A}_{1}=0$ to the Euler-Lagrange equations in an ad hoc manner.

Second, the Maxwell equations for $A_{A}$ have the additional term involving $N_{A B}$. If it happens that $N_{A B}$ is symmetric, then a Lagrangian can be found to reproduce this term: The Lagrangian would add the term $\frac{1}{2} A_{A} A_{B} N_{A B}$ to the reduced Lagrangian equation (B14). However, if $N_{A B}$ is not symmet- 
ric, then such a Lagrangian would not in general be possible-certainly no Lagrangian could be found to reproduce the Maxwell equations exactly, though under some circumstances it may be possible to find a Lagrangian which would produce equations equivalent to the Maxwell equations.

For example, the standard structure constants of a Bianchi type III model [1] are $C_{12}^{2}=-C_{21}^{2}=1$, the other structure constants vanishing. In this case $N_{A B}=\operatorname{diag}(1,0)$, and the Maxwell equations can indeed be derived from a variational principle (with the constraint $0=\dot{A}_{1}$ being put in by hand): The Maxwell equations are

$$
0=\dot{A}_{1}=\ddot{A}_{1}=\ddot{A}_{2}=\ddot{A}_{3},
$$

which clearly can come from a constrained variational principle. Note that in this type III case the reduced Lagrangian yields as Euler-Lagrange equations

$$
0=\ddot{A}_{1}=\ddot{A}_{2}+A_{2}=\ddot{A}_{3},
$$

which are not at all the same as the Maxwell equations.

A second example is that of a group which has structure constants $C_{12}^{2}=-C_{21}^{2}=C_{13}^{2}=-C_{31}^{2}=1$, the rest being zero. In fact, this group is also type III, in a basis which is a linear transformation of the basis in the preceding example. Since this transformation is not an orthogonal one, the Maxwell equations in this case differ significantly from the preceding. The reduced Lagrangian is

$$
\mathcal{L}_{R}=-\frac{1}{2}\left(\dot{A}_{1}^{2}+\dot{A}_{2}^{2}+\dot{A}_{2}^{2}\right)+A_{2}^{2} .
$$

The Euler-Lagrange equations are

$$
0=\ddot{A}_{1}=\ddot{A}_{2}+2 A_{2}=\ddot{A}_{3} \text {. }
$$

The Maxwell equations are

$$
0=\dot{A}_{1}=\ddot{A}_{1}=\ddot{A}_{2}=\ddot{A}_{3}-A_{2} .
$$

The difference between the Maxwell equations and the Euler-Lagrange equations in this example are profound: First, the Maxwell equations include the constraint $\dot{A}_{1}=0$, as in the previous example. Second, the Maxwell equations cannot be derived from a variational principle, unlike the previous example.

\section{APPENDIX C: RELATIONSHIP BETWEEN SETTING THE GAUGE AND VARIATIONAL PRINCIPLES}

Here we summarize and expand the main result of [14]. For simplicity we will use the language of mechanics (a finite number of degrees of freedom), although everything can be translated to field theory. Consider a singular Lagrangian $L$ that leads to some primary constraints, $\phi_{\rho}=0$ in phase space, presumed to be effective (each has a non-vanishing gradient on the constraint surface). For the sake of simplicity we will consider the case when all the constraints, primary, secondary, etc., are first class (this is what happens in our generally covariant theories). The equations of motion obtained from $L$ are

$$
\frac{\delta L}{\delta q^{i}}=\alpha_{i}-W_{i j} \ddot{q}^{j}=0
$$

where

$$
W_{i j} \ddot{q}^{j}=\frac{\partial^{2} L}{\partial \dot{q}^{i} \dot{q}^{j}} \quad \text { and } \quad \alpha_{i}=\frac{\partial L}{\partial q^{i}}-\frac{\partial^{2} L}{\partial \dot{q}^{i} \partial q^{j}} \dot{q}^{j}-\frac{\partial^{2} L}{\partial \dot{q}^{i} \partial t} .
$$

The Lagrangian dynamics can equivalently be described by a vector field that exists on, and is tangent to, the constraint surface in configuration-velocity space:

$$
\mathbf{X} \equiv \frac{\partial}{\partial t}+\dot{q}^{i} \frac{\partial}{\partial q^{i}}+a^{i}(q, \dot{q}) \frac{\partial}{\partial \dot{q}^{i}}+\eta^{\rho} \boldsymbol{\Gamma}_{\rho} \equiv \mathbf{X}_{0}+\eta^{\rho} \boldsymbol{\Gamma}_{\rho} .
$$

The $a^{i}$ are determined from the equations of motion and the stabilization algorithm, $\eta^{\rho}$ are arbitrary functions of time (or spacetime in field theory) and any other variable, and $\boldsymbol{\Gamma}_{\rho}$ is

$$
\boldsymbol{\Gamma}_{\rho}=\gamma_{\rho}^{i} \frac{\partial}{\partial \dot{q}^{i}},
$$

where $\gamma_{\rho}^{i}$ are the null vectors of $W_{i j}$. These null vectors can be given as

$$
\gamma_{\rho}^{i}=\frac{\partial \phi_{\rho}}{\partial p_{i}}(q, \hat{p}),
$$

where $\hat{p}_{i}(q, \dot{q})=\partial L / \partial \dot{q}^{i}$. Let us point out that $\eta^{\rho} \boldsymbol{\Gamma}_{\rho}$ in Eq. (C1) is the piece that corresponds in our case to Eq. (4.1).

Notice that $\alpha_{i} \gamma_{\rho}^{i}=0$ is a consequence of the equations of motion $\delta L / \delta q^{i}=0$. They are called the primary Lagrangian constraints.

Consider now a partial gauge fixing of the dynamics given by a set of new constraints $\chi_{\rho^{\prime}}=0$, with $\left|\rho^{\prime}\right|<|\rho|$, defined in configuration space (holonomic constraints). Let us split the set of indices $\rho$ into two sets, $\rho^{\prime}$ and $\rho^{\prime \prime}$, in such a way that $\boldsymbol{\Gamma}_{\rho^{\prime \prime}} \dot{\chi}_{\sigma^{\prime}}=0$ and $\left|\boldsymbol{\Gamma}_{\rho^{\prime}} \dot{\chi}_{\sigma^{\prime}}\right| \neq 0$. Then, the requirement $\mathbf{X}\left(\dot{\chi}_{\rho^{\prime}}\right)=0$ determines the functions $\eta^{\rho^{\prime}}$ and leaves completely undetermined $\eta^{\rho^{\prime \prime}}$.

In such a situation, the following result is proved in [14]: If we plug the holonomic gauge fixing $\chi_{\rho^{\prime}}=0$ into the original Lagrangian $L$ to get the reduced Lagrangian $L_{\mathrm{GF}}$, then the following equivalence holds (where $[L]$ is the EulerLagrange variation):

$$
[L]=0, \quad \chi_{\rho^{\prime}}=0 \Leftrightarrow\left[L_{\mathrm{GF}}\right]=0, \quad \alpha_{i} \gamma_{\rho^{\prime}}^{i}=0,
$$

where $\alpha_{i} \gamma_{\rho^{\prime}}^{i}$ are a subset of the primary Lagrangian constraints for $L$ with the understanding that the gauge fixing has been plugged into them.

So we see that to describe the same motions in the reduced space it is not enough in general to impose the new 
Lagrangian equations of motion, $\left[L_{\mathrm{GF}}\right]=0$, but some additional constraints must be required, too.

Now, we will prove, using the machinery of [14], that in order for the solutions of $\left[L_{\mathrm{GF}}\right]=0$ to satisfy the constraints $\alpha_{i} \gamma_{\rho^{\prime}}^{i}=0$, we only need to impose them in the initial conditions.

Since the gauge-fixing constraints $\chi_{\rho^{\prime}}=0$ are holonomic, they just reduce the configuration space. We have adopted the notation $q^{i}$ for the local coordinates in the original configuration space. We will adopt the notation $Q^{a}$ for the local coordinates in the reduced configuration space.

It is proved in [14] that the Lagrangian evolution operator in the reduced velocity space takes the form

$$
\begin{aligned}
\mathbf{X}_{\mathrm{R}} & \equiv \frac{\partial}{\partial t}+\dot{Q}^{a} \frac{\partial}{\partial Q^{a}}+a^{a}(Q, \dot{Q}) \frac{\partial}{\partial \dot{Q}^{a}}+\widetilde{\eta}^{\rho^{\prime \prime}} \widetilde{\boldsymbol{\Gamma}}_{\rho^{\prime \prime}} \\
& \equiv \mathbf{X}_{\mathrm{R}_{0}}+\widetilde{\eta}^{\rho^{\prime \prime}} \widetilde{\boldsymbol{\Gamma}}_{\rho^{\prime \prime}},
\end{aligned}
$$

where $\widetilde{\eta}^{\rho^{\prime \prime}}$ are arbitrary functions; $\widetilde{\boldsymbol{\Gamma}}_{\rho^{\prime \prime}}$ is

$$
\widetilde{\boldsymbol{\Gamma}}_{\rho^{\prime \prime}}=\widetilde{\gamma}_{\rho^{\prime \prime}}^{a} \frac{\partial}{\partial \dot{Q}^{a}},
$$

and similarly to Eq. (C2), $\tilde{\gamma}_{\rho^{\prime \prime}}^{a}$ is defined by

$$
\widetilde{\gamma}_{\rho^{\prime \prime}}^{a}=\frac{\partial \widetilde{\phi}_{\rho^{\prime \prime}}}{\partial P_{a}}(Q, \hat{P}),
$$

where $\widetilde{\phi}_{\rho^{\prime \prime}}$ are the primary Hamiltonian constraints corresponding to the reduced theory. It turns out [14] that these constraints are related to the original constraints $\phi_{\rho^{\prime \prime}}$ by

$$
\phi_{\rho^{\prime \prime}}(q(Q), p)=\widetilde{\phi}_{\rho^{\prime \prime}}\left(Q, p \frac{\partial q}{\partial Q}\right) .
$$

From all these results, the following equalities hold:

$$
\begin{aligned}
\widetilde{\boldsymbol{\Gamma}}_{\rho^{\prime \prime}}\left(\left.\left(\alpha_{i} \gamma_{\rho^{\prime}}^{i}\right)\right|_{q(Q)}\right) & =\frac{\partial \widetilde{\phi}_{\rho^{\prime \prime}}^{a}}{\partial P_{a}} \frac{\partial\left(\left.\left(\alpha_{i} \gamma_{\rho^{\prime}}^{i}\right)\right|_{q(Q)}\right)}{\partial \dot{Q}^{a}} \\
& =\left.\left(\boldsymbol{\Gamma}_{\rho^{\prime \prime}}\left(\alpha_{i} \gamma_{\rho^{\prime}}^{i}\right)\right)\right|_{q(Q)} \\
& =\left.\mathcal{F} L^{*}\left\{\phi_{\rho^{\prime}}, \phi_{\rho^{\prime \prime}}\right\}\right|_{q(Q)}=0 .
\end{aligned}
$$

Here $\mathcal{F} L^{*}$ is the pullback of the Legendre map $\mathcal{F} L$ from velocity space to phase space. We have used in the last equality the fact that the pullback of a primary Hamiltonian constraint is identically zero.

With this new result, $\widetilde{\boldsymbol{\Gamma}}_{\rho^{\prime \prime}}\left(\left.\left(\alpha_{i} \gamma_{\rho^{\prime}}^{i}\right)\right|_{q(Q)}\right)=0$, we can see that the arbitrary part in the reduced Lagrangian evolution operator equation $(\mathrm{C} 4)$ has no effect on $\left.\left(\alpha_{i} \gamma_{\rho^{\prime}}^{i}\right)\right|_{q(Q)}$. This means that in the constraint surface for the reduced theory (this surface is determined by the equations of motion for $\left.L_{\mathrm{GF}}\right)$, the operator $\mathbf{X}_{\mathrm{R}_{0}}$ must also be tangent to the surface $\left(\left.\left(\alpha_{i} \gamma_{\rho^{\prime}}^{i}\right)\right|_{q(Q)}\right)=0$. [This is the only way to ensure the equivalence in Eq. (C3), for if the operator $\mathbf{X}_{\mathrm{R}_{0}}$ is not tangent to the surface $\left(\left.\left(\alpha_{i} \gamma_{\rho^{\prime}}^{i}\right)\right|_{q(Q)}\right)=0$, then there will be no solutions of the equations of motion for the original Lagrangian in the gauge $\chi_{\rho^{\prime}}=0$, and we know that these solutions exist.] Therefore, if we consider a solution of $\left[L_{\mathrm{GF}}\right]=0$ with initial conditions satisfying $\left.\left(\alpha_{i} \gamma_{\rho^{\prime}}^{i}\right)\right|_{q(Q)}=0$, then the whole solution satisfies $\left.\left(\alpha_{i} \gamma_{\rho^{\prime}}^{i}\right)\right|_{q(Q)}=0$.

\section{APPENDIX D: DEGREES OF FREEDOM}

In this appendix we illustrate with a very simple case the counting of degrees of freedom for our models. We also discuss the fact that spacetime rigid symmetries may be understood as residual gauge transformations.

We start by counting the true degrees of freedom for the Bianchi type I case. The counting can be done in configuration-velocity space or in phase space-the counting is the same [13]. We start with the variables $N$ and $\dot{N}, N^{a}$ and $\dot{N}^{a}, g_{a b}$ and $\dot{g}_{a b}: 20$ apparent degrees of freedom. The shift vector variables may be eliminated by our general considerations, so that 14 apparent degrees of freedom are left.

There is one constraint in the Lagrangian formalism. To fix the time reparametrization invariance we define the time parameter as a function of our dynamical variables, excluding $N$. Its stability, that is, the fact that the time derivative of this definition must vanish, will give a new constraint, containing the variable $N$, with no time dependence. The stability of this last constraint gives a new constraint that can be used to isolate $\dot{N}$. The requirement of stability now determines the arbitrary function in the Lagrangian evolution operator, and no more constraints appear. We are left with $1+3$ constraints (one true constraint plus three gauge fixing constraints), lowering the number of degrees of freedom to 10. The same counting can be done in phase space: In this case, there are 2 true constraints, and we must introduce 2 gauge fixing constraints.

In type I the momentum constraints are identically zero, and so they do not reduce the degrees of freedom. We are only left with HPD symmetries. Every constant matrix $B_{b}^{a}$ of Eq. (7.16) defines an automorphism of the Lie algebra.

Now topology enters the picture. If the topology of $\Sigma$ is " "open", (for example, $\mathbb{R}^{3}$ ), simply connected, and with global Killing vectors, then to each matrix $B_{b}^{a}$ there corresponds an HPD through Eq. (5.2). Therefore, there are nine HPD gauge degrees of freedom. The generators of HPDs, Eq. (7.17), are not constraints, because in this case Eqs. (7.11) and (7.14) differ by boundary terms. So the final number of degrees of freedom for a Bianchi type I model with surfaces of homogeneity with the topology of $\mathbb{R}^{3}$ is $10-9=1$.

It may appear strange to some people that a Hamiltonian formalism can turn out to have an odd number of degrees of freedom, as in the above example. In fact, we have already said that HPDs need only be implemented in the initial conditions, and so in this sense we have a Hamiltonian formulation plus an equivalence relation coming from the outset: It is not generated by the reduced formalism but is a remnant of the generally covariant theory we started with. To find the 
number of degrees of freedom number is a matter of counting constraints, which trajectories count as the "same" as others, and whether a parametrized trajectory or its orbit (its one-dimensional set of points in phase space) should be considered as physically significant. We have been guided by the principle that the Lagrangian and Hamiltonian formalisms should be equivalent (see [13]) in coming to the conclusion that in fact they are.

If the topology of $\Sigma$ is that of a three-torus, then there are no infinitesimal HPDs available (there are finite HPDs but they do not reduce local degrees of freedom). We end up with a final number of 10 degrees of freedom. We will not consider other topologies.

Let us turn to the second consideration in this appendix, the possibility of interpreting rigid spacetime symmetries as residual gauge transformations. Consider a free particle in $\mathrm{R}^{4}$ in a gravitational background. The action is

$$
S=\int\left(g_{\mu \nu} \dot{x}^{\mu} \dot{x}^{\nu}\right)^{1 / 2} d \tau .
$$

What are the degrees of freedom? If we start with a generally covariant theory, we know that diffeomorphisms correspond to gauge degrees of freedom. Let us consider the metric background as non-dynamical, take the passive view for the action of diffeomorphisms, and consider the simplified case where there exists a system of spacetime coordinates such that the metric is just a Minkowski metric. Now we can make the following gauge fixing: We decide to stick with Minkowskian coordinates and only allow further diffeomorphisms if they keep this condition. We end up with Poincaré transformations as residual gauge transformations.

To count the degrees of freedom, we start with positions $x^{\mu}$ and velocities $\dot{x}^{\mu}$, which amount to 8 degrees of freedom.
To fix the $\tau$ reparametrization invariance we must spend two constraints (for instance $x^{0}-\tau=0$ and its stabilization $\dot{x}^{0}$ $=1)$. We are left with 6 degrees of freedom. The residual gauge freedom consists of Lorentz transformations, but our gauge fixing forbids boosts and time translations, and so we are left with three-translations and rotations. Rotations only affect 2 degrees of freedom, because the norm of the velocity three-vector is unchanged, and therefore we eliminate 5 of the 6 remaining degrees of freedom. We end up with a single degree of freedom that in our gauge fixing corresponds to the kinetic energy.

Here we see a matter of interpretation as to what is and what is not a true degree of freedom. From our point of view, if the Lorentz invariance may be considered as the residual invariance found after a process of reducing the gauge group of general covariance, then the Lorentz degrees of freedom must be considered as gauge degrees of freedom. But as residual gauge symmetries, they are not associated with constraints but to ordinary constants of motion.

Summing up, the analysis of degrees of freedom, either in the case of Bianchi cosmologies or the simple case of a Minkowskian free particle, depends upon the point of view adopted. If one sticks to the formalism by itself, that is to say, to what the given Lagrangian yields as constraints, gauge transformations, and so on, one does not get the same number of degrees of freedom as when one considers that our theory comes from the reduction of an originally generally covariant theory. In this second point of view (which is the one we support) there are some symmetry transformations that are residual gauge transformations, that is, remnants of the reduction procedure of the Lagrangian and the gauge group, which require gauge fixing.
[1] A. H. Taub, Ann. Math. 53, 472 (1951).

[2] G. F. R. Ellis and M. A. H. MacCallum, Commun. Math. Phys. 12, 108 (1969).

[3] S. W. Hawking, Mon. Not. R. Astron. Soc. 142, 129 (1969).

[4] M. A. H. MacCallum, Commun. Math. Phys. 20, 57 (1971).

[5] M. A. H. MacCallum and A. H. Taub, Commun. Math. Phys. 25, 173 (1972).

[6] M. P. Ryan, J. Math. Phys. 15, 812 (1974).

[7] G. E. Sneddon, J. Phys. A 9, 229 (1976).

[8] M. A. H. MacCallum, in General Relativity: An Einstein Centenary Survey, edited by S. W. Hawking and W. Israel (Cambridge University Press, Cambridge, England, 1979), Chap. 11.

[9] A. Ashtekar and J. Samuel, Class. Quantum Grav. 8, 2191 (1991).

[10] R. T. Jantzen, Commun. Math. Phys. 64, 211 (1979).

[11] R. M. Wald, General Relativity (University of Chicago Press, Chicago, 1984).
[12] C. Batlle, J. Gomis, J. M. Pons, and N. Roman-Roy, J. Math. Phys. 27, 2953 (1986).

[13] J. M. Pons and L. C. Shepley, Class. Quantum Grav. 12, 1771 (1995).

[14] J. M. Pons, Int. J. Mod. Phys. A 11, 975 (1996).

[15] M. P. Ryan and L. C. Shepley, Homogeneous Relativistic Cosmologies (Princeton University Press, Princeton, 1975).

[16] T. Koike, M. Tanimoto, and A. Hosoya, J. Math. Phys. 35, 4855 (1994).

[17] T. Koike, M. Tanimoto, and A. Hosoya, J. Math. Phys. 38, 350 (1997).

[18] M. Tanimoto, T. Koike, and A. Hosoya, J. Math. Phys. 38, 6560 (1997).

[19] J. M. Pons, D. C. Salisbury, and L. C. Shepley, Phys. Rev. D 55, 658 (1997).

[20] O. Coussaert and M. Henneaux, Class. Quantum Grav. 10, 1607 (1993). 